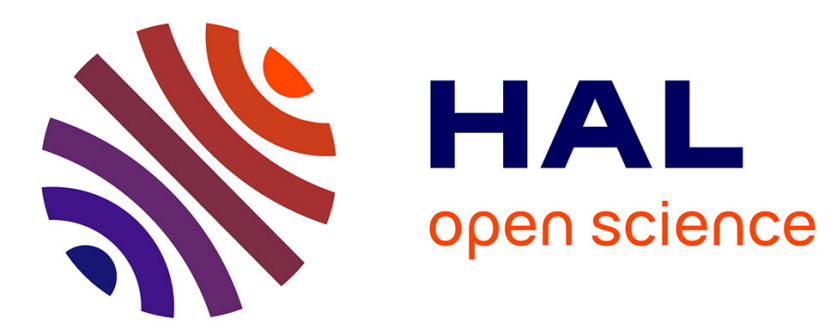

\title{
Classical isomorphisms of types
}

Olivier Laurent

\section{To cite this version:}

Olivier Laurent. Classical isomorphisms of types. Mathematical Structures in Computer Science, 2005, 15, pp.969-1004. 10.1017/S0960129505004895 . hal-00003761

\section{HAL Id: hal-00003761 https://hal.science/hal-00003761}

Submitted on 4 Jan 2005

HAL is a multi-disciplinary open access archive for the deposit and dissemination of scientific research documents, whether they are published or not. The documents may come from teaching and research institutions in France or abroad, or from public or private research centers.
L'archive ouverte pluridisciplinaire HAL, est destinée au dépôt et à la diffusion de documents scientifiques de niveau recherche, publiés ou non, émanant des établissements d'enseignement et de recherche français ou étrangers, des laboratoires publics ou privés. 


\title{
Classical isomorphisms of types
}

\author{
Olivier LAURENT \\ Preuves, Programmes et Systèmes \\ CNRS - Université Paris VII \\ UMR 7126 - Case 7014 \\ 175, rue du Chevaleret - 75013 Paris - FRANCE \\ Olivier.Laurent@pps.jussieu.fr
}

April 4, 2003

\begin{abstract}
The study of isomorphisms of types has been mainly carried out in an intuitionistic setting. We extend some of these works to classical logic for both call-by-name and call-by-value computations by means of polarized linear logic and game semantics. This leads to equational characterizations of these isomorphisms for all the propositional connectives.
\end{abstract}

\section{Introduction}

The study of isomorphisms of types started from theoretical questions about the characterization of isomorphisms in free cartesian closed categories [23] and in the $\lambda$-calculus $[11,8]$. The possibility of using these results as a basis for more practical tools (programming library search [21], gluing of software components, ...) has considerably increased the interest of people on the subject.

The underlying problem is the following: given the definition of isomorphisms of types,

\section{Definition 1 (Isomorphims of types)}

Let $\mathcal{S}$ be a logical system, with an equational theory $=\mathcal{S}$ on proofs, two formulas $A$ and $B$ are isomorphic, denoted by $A \simeq_{\mathcal{S}} B$, if there exist two proofs $\pi_{A}$ of $B \vdash A$ and $\pi_{B}$ of $A \vdash B$ such that by cutting $\pi_{A}$ and $\pi_{B}$ on the conclusion $B$ (resp. $A$ ) we obtain a proof equal, up to $=\mathcal{S}$, to the axiom $A \vdash A$ (resp. $B \vdash B$ ).

is it possible to find an equational theory corresponding exactly to the isomorphisms of the system $\mathcal{S} ?$

Many works have been done for characterizing these isomorphisms of types for fragments of the propositional $\lambda$-calculus, second order $\lambda$-calculus, extensions with recursive types, with dependent types, ... However all these works have been carried out in an intuitionistic setting. Our main goal is to give a first attempt in the extension of these results to a classical setting.

While the question of the study of intuitionistic isomorphisms of types seems clear to formulate, the classical case is not so simple. There are mainly three different possible questions:

- what are the isomorphisms in a non deterministic classical setting (such as LK)?

- what are the isomorphisms in a call-by-name classical setting? 
- what are the isomorphisms in a call-by-value classical setting?

We are going to address these three questions in a propositional setting with all the classical connectives. The first question is mainly degenerated and doesn't give an interesting notion of isomorphisms. In order to solve the two other questions, we will study the isomorphisms of the polarized variant of linear logic LLP [15] because both call-by-name and call-by-value classical logics are embedded in LLP through syntactical reduction-preserving translations. This entails that, from the characterization of isomorphisms in LLP, we can deduce both call-by-name and call-by-value isomorphisms.

For the study of the isomorphisms of LLP, we use a semantical approach. Such an approach consists in finding a denotational model of the system (so that any syntactical isomorphism leads to an isomorphism in the model) in which first the isomorphisms are possible to compute and second there are no more isomorphisms in the model than in the syntax. For that purpose, we will use game semantics which is known to give precise models of the syntax (full abstraction and full completeness results) and makes computations easier than in the syntax. This gives an application of game semantics to a purely syntactical question, showing that even if games seem very near to the syntax they are however abstract enough to simplify computations. The main consequences are two different characterizations of the polarized isomorphisms: an equational theory and a graphical representation through polarized forests.

We have tried to organize the paper by putting in different sections the intermediate results that use different techniques in such a way that the reader only interested in a particular topic could understand it by only reading the statements of the final results of the previous sections.

Section 1 is a short section devoted to solve our first question about non-deterministic classical isomorphisms of types. The other sections only look at the deterministic case.

Section 2 contains the syntactical definition of polarized linear logic (LLP) which is the main logical system used in the following sections. Section 3 gives some elements of game semantics and proves the key result of the paper which is the characterization of the isomorphisms in the game model. Section 4 defines the interpretation of LLP in the game model showing that any isomorphism in LLP leads to an isomorphism in games. Section 5 relates isomorphisms in the game model and isomorphisms in LLP (without using games anymore) by computing the corresponding equational theory and by showing that all the obtained isomorphisms are syntactically correct. This ends the study of isomorphisms in LLP. Section 6 uses embeddings of the call-by-name $\lambda \mu$-calculus and of the call-by-value $\lambda \mu$-calculus into LLP to derive the corresponding equational theories of isomorphisms. As a consequence, we get back the intuitionistic case.

Section 7 explains the relation between our classical results on isomorphisms with disjunction and the intuitionistic ones of Balat-Di Cosmo-Fiore [13] by showing that they are in fact not really possible to compare due to different underlying theories of the disjunction. Section 8 presents a relation between our classical theory of isomorphisms and the equations of the real exponential field $(\mathbb{R},+, 0, \cdot, 1, e)$ in the spirit of Tarski's problems for natural numbers studied in the usual theory of isomorphisms of types.

\section{Non deterministic isomorphisms}

In this very short section, we just want to close quickly the question of isomorphisms in the non deterministic classical setting by proving that it is degenerated. We prove that this non determinism entails that any two equiprovable formulas are isomorphic due to the too strong equational theory on proofs generated by cut elimination (any two proofs of a given sequent are equal). 
We consider proofs in Gentzen's sequent calculus LK up to cut elimination and:

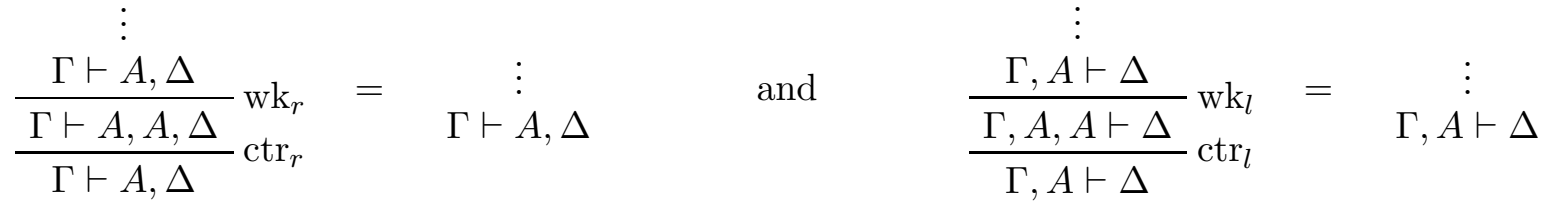

Proposition 1 (Isomorphisms in LK)

$A \dashv \vdash_{\mathrm{LK}} B$ if and only if $A \simeq_{\mathrm{LK}} B$.

Proof: The second direction is immediate. For the first one, from $A \vdash_{\mathrm{LK}} B$ and $B \vdash_{\mathrm{LK}} A$ we can deduce a proof $\pi_{A}$ of $A \vdash_{\mathrm{LK}} A$ and a proof $\pi_{B}$ of $B \vdash_{\mathrm{LK}} B$, but using Lafont's critical pair, these two proofs are equal to the identity:

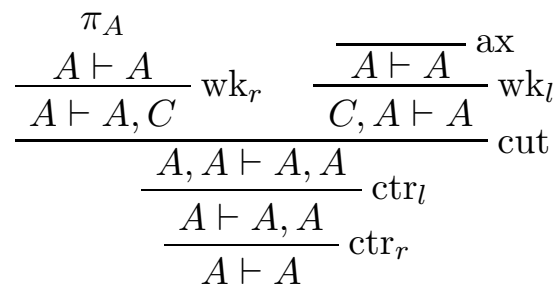

reduces to both $\pi_{A}$ and the identity axiom.

This explains why we have to go to some more constrained systems to get interesting theories of isomorphisms.

\section{Polarized Linear Logic}

Polarized linear logic (LLP) is based on a restriction of the formulas of linear logic in order to get a simpler system rich enough to interpret the $\lambda$-calculus and classical logic. Polarized formulas are given by the following grammar:

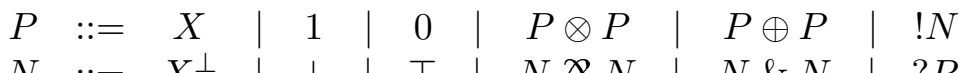

$$
\begin{aligned}
& N::=X^{\perp}|\perp| \top|N \not 8 N| N \& N \mid \quad ? P
\end{aligned}
$$

The orthogonal of a formula is defined by the De Morgan's laws:

$$
\begin{array}{rlrrrrl}
(X)^{\perp} & = & X^{\perp} & \left(X^{\perp}\right)^{\perp} & = & X \\
\perp^{\perp} & = & 1 & 1^{\perp} & = & \perp \\
\top^{\perp} & = & 0 & 0^{\perp} & = & \top \\
(N \ngtr M)^{\perp} & = & N^{\perp} \otimes M^{\perp} & (P \otimes Q)^{\perp} & = & P^{\perp} \ngtr Q^{\perp} \\
(N \& M)^{\perp} & = & N^{\perp} \oplus M^{\perp} & (P \oplus Q)^{\perp} & = & P^{\perp} \& Q^{\perp} \\
(? P)^{\perp} & = & ! P^{\perp} & (! N)^{\perp} & = & ? N^{\perp}
\end{array}
$$

and for any polarized formula $A,\left(A^{\perp}\right)^{\perp}=A$.

The rules of LLP are not just the rules of LL restricted to polarized formulas otherwise classical logic would not be so easy to embed. This is why we also generalize structural rules (?w, ?c and 
context of !) to any negative formula and not only ?-formulas.

$$
\begin{array}{cc}
\frac{\vdash N, N^{\perp}}{\vdash x} & \frac{\vdash \Gamma, N \quad N^{\perp}, \Delta}{\vdash \Gamma, \Delta} \text { cut } \\
\frac{\vdash \Gamma, N, M}{\vdash \Gamma, N \gamma M} \text { \& } & \frac{\vdash \Gamma, P \quad \vdash \Delta, Q}{\vdash \Gamma, \Delta, P \otimes Q} \otimes \\
\frac{\vdash \Gamma, N \quad \vdash \Gamma, M}{\vdash \Gamma, N \& M} \& & \frac{\vdash \Gamma, P}{\vdash \Gamma, P \oplus Q} \oplus_{1} \frac{\vdash \Gamma, Q}{\vdash \Gamma, P \oplus Q} \oplus_{2} \\
\frac{\vdash \mathcal{N}, N}{\vdash \mathcal{N}, ! N} ! \quad \frac{\vdash \Gamma, P}{\vdash \Gamma, ? P} ? d \frac{\vdash \Gamma}{\vdash \Gamma, N} ? w \frac{\vdash \Gamma, N, N}{\vdash \Gamma, N} ? c \\
\frac{\vdash \Gamma, \top}{\vdash} \frac{\frac{\vdash \Gamma}{\vdash \Gamma, \perp} \perp}{\vdash 1} 1
\end{array}
$$

The context of the $T$-rule must contain at most one positive formula and $\mathcal{N}$ is a context of negative formulas.

\section{Lemma 1 (Positive formula)}

If $\vdash \Gamma$ is provable in LLP, $\Gamma$ contains at most one positive formula.

The equational theory we consider on LLP proofs, denoted by $\pi_{1}={ }_{\beta \eta} \pi_{2}$, is given by cutelimination, $\eta$-expansion of axioms and the following equations:

$$
\begin{aligned}
& \underset{\vdash \Gamma, \top}{\vdots}=\overline{\vdash \Gamma, \top} \top \\
& \begin{array}{cc}
\frac{\vdash \Gamma, N}{\vdash \Gamma, N, N} ? w \\
\frac{\vdash \Gamma, N}{\vdash} c & \vdots
\end{array}
\end{aligned}
$$

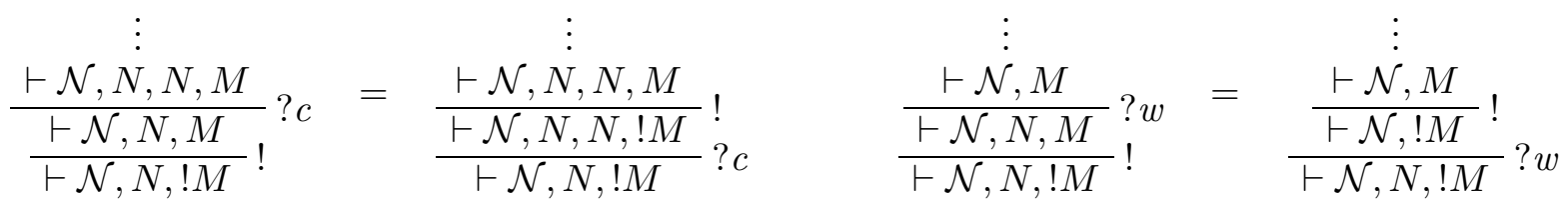

\section{Definition 2 (Polarized isomorphim)}

Let $A$ and $B$ be two polarized formulas, $A$ and $B$ are isomorphic, denoted by $A \simeq_{\mathrm{LLP}} B$, if there exist two proofs $\pi_{A}$ of $\vdash B^{\perp}, A$ and $\pi_{B}$ of $\vdash A^{\perp}, B$ such that by cutting $\pi_{A}$ and $\pi_{B}$ on the conclusion $B$ (resp. $A$ ) we obtain a proof equal to the axiom $\vdash A^{\perp}, A\left(\right.$ resp. $\left.\vdash B^{\perp}, B\right)$, up to $=_{\beta \eta}$.

\section{Definition 3 ( $\mathcal{E}$-isomorphism)}

Let $\mathcal{E}$ be the equational theory generated by the following set of equations (for all negative formulas 
$N, M$ and $L)$ :

$$
\begin{aligned}
N \not 8 M & =M \not 8 N \\
(N \not 8) \not 8 L & =N \not 8(M \not 8 L) \\
N \not 8 \perp & =N \\
N \& M & =M \& N \\
(N \& M) \& L & =N \&(M \& L) \\
N \& \top & =N \\
N \not 8(M \& L) & =(N \not 8 M) \&(N \not 8 L) \\
N \not \supset \top & =\top \\
!(N \& M) & =! N \otimes ! M \\
! \top & =1
\end{aligned}
$$

together with the dual ones.

Let $A$ and $B$ be two polarized formulas, $A$ and $B$ are $\mathcal{E}$-isomorphic, denoted by $A \simeq_{\mathcal{E}} B$, if they are equal in the theory $\mathcal{E}$.

\section{Lemma 2 (Correctness of $\mathcal{E}$ )}

If $A \simeq_{\mathcal{E}} B$ then $A \simeq_{\mathrm{LLP}} B$.

Proof: The proofs corresponding to the equations are:

$$
\begin{aligned}
& \frac{\overline{\vdash N^{\perp}, N} \text { ax } \frac{\vdash M^{\perp}, M}{\vdash N^{\perp} \otimes M^{\perp}, N, M}}{\frac{\vdash N^{\perp} \otimes M^{\perp}, M}{\vdash}{ }^{\perp} N} \otimes
\end{aligned}
$$

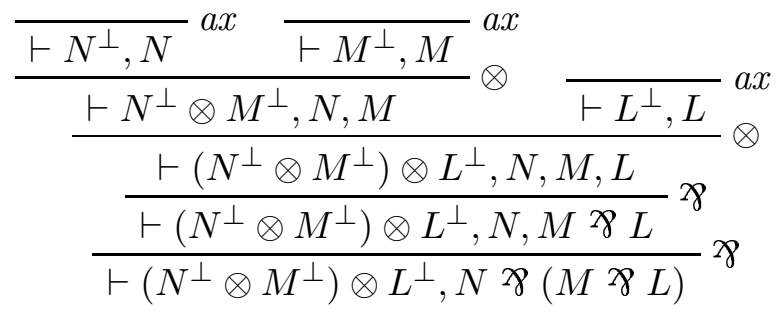

$$
\begin{aligned}
& \frac{\overline{\vdash N}^{\perp}, N}{\vdash N^{\perp} \otimes 1, N} \quad \overline{\vdash 1}^{1} \otimes \\
& \frac{\frac{{ }^{\perp} N^{\perp}, N}{\vdash N^{\perp}, N, \perp} \perp}{\vdash N^{\perp}, N \not 8 \perp} \not 8 \\
& \frac{\overline{\vdash N, \top, 0}^{\top} \text { \& }}{\vdash N^{\gamma \top}, 0} \quad \overline{\vdash N^{\perp} \otimes 0, \top}{ }^{\top}
\end{aligned}
$$




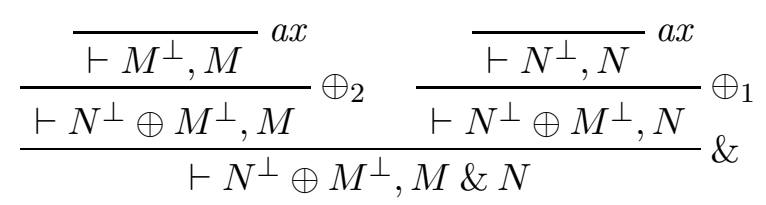

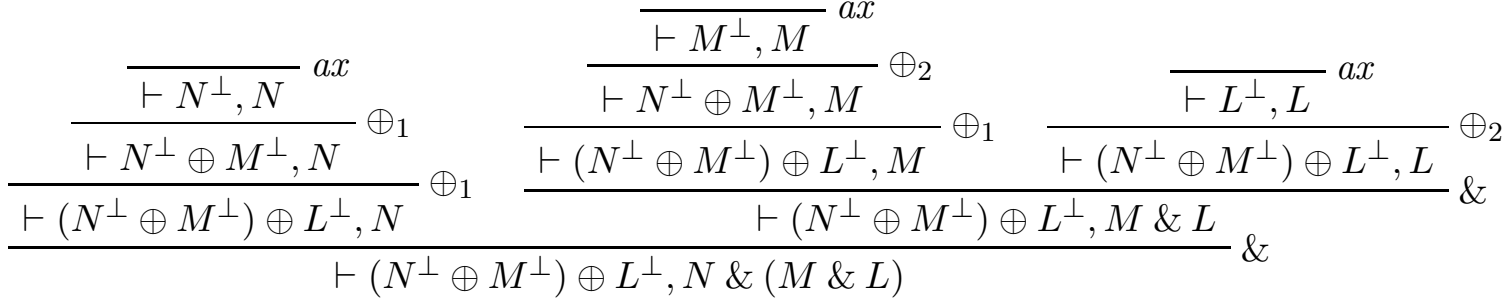

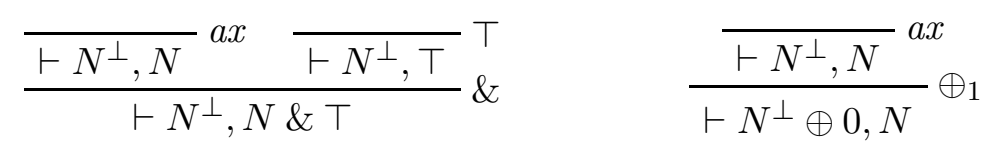

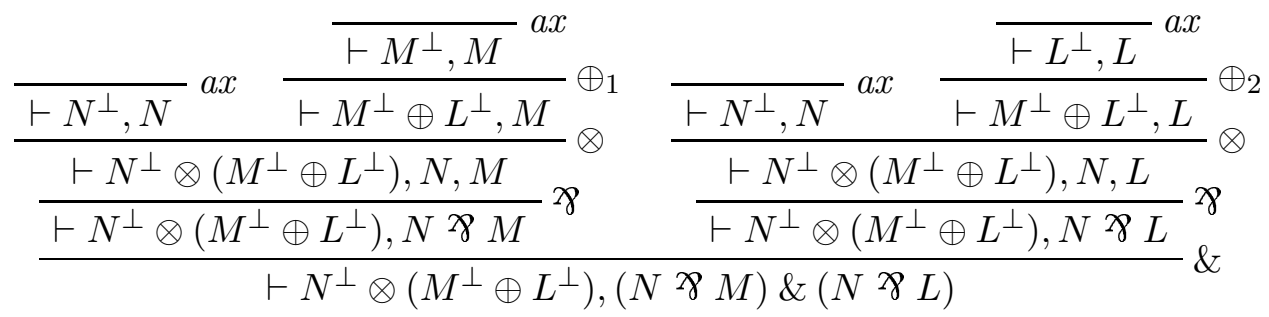

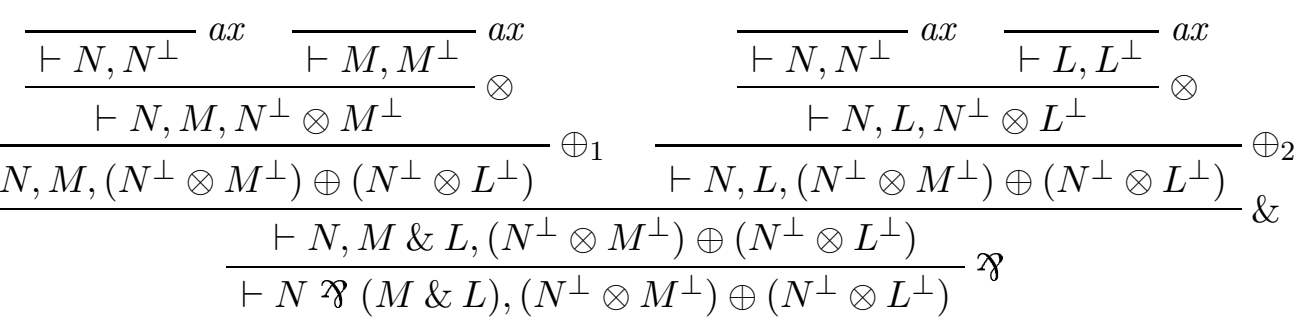

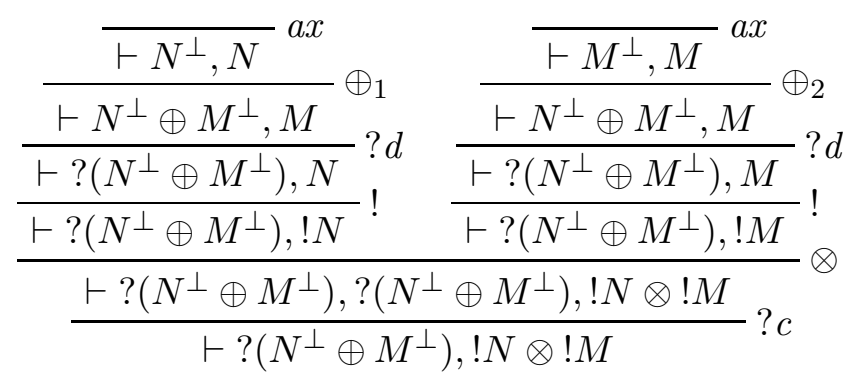

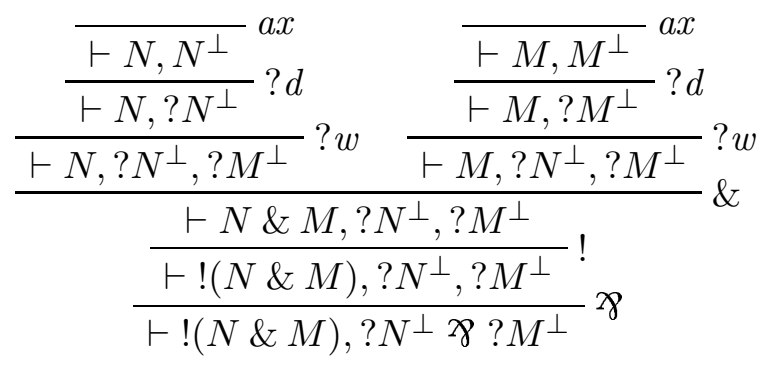




$$
\frac{\overline{\vdash \top, \perp} \top}{\vdash ! \top, \perp} ! \quad \frac{{ }^{1}}{\vdash ? 0,1} ? w
$$

We then have to verify that, up to $=_{\beta \eta}$, cutting the two corresponding proofs gives an axiom.

\section{Game semantics}

Game semantics gives denotational models for various systems. We will use it because it is far enough from the syntax to simplify computations and not too much to betray the syntax. Types are interpreted by arenas (forests) and proofs or programs by strategies on these arenas.

\subsection{Arenas and strategies}

We just recall the key definitions of usual game semantics. All these definitions with more details, explanations and justifications appear in [14].

\section{Definition 4 (Forest)}

A forest is a partial order $(E, \leq)$ such that if $x \leq z$ and $y \leq z$ then either $x \leq y$ or $y \leq x$.

The nodes of a forest $(E, \leq)$ are the elements of $E$ and the forest is finite if $E$ is finite. The roots are the minimal elements. If $x$ is the maximal element under $y$, we say that $y$ is a son of $x$, denoted by $x<^{1} y$. If $(E, \leq)$ is a forest, the relation $\leq$ is the transitive reflexive closure of $<^{1}$ and we will sometimes represent $(E, \leq)$ by $\left(E,<^{1}\right)$.

\section{Definition 5 (Morphism)}

A morphism between two forests $(E, \leq)$ and $\left(E^{\prime}, \leq^{\prime}\right)$ is a function from $E$ to $E^{\prime}$ which respects the order (if $x \leq y$ then $f(x) \leq^{\prime} f(y)$ ).

If there exists a forest isomorphism between $E$ and $E^{\prime}$, we use the notation $E \simeq_{f} E^{\prime}$.

\section{Definition 6 (Arena)}

An arena $A$ is a finite forest whose nodes are called moves.

The polarity $\lambda_{A}(a)$ of a move $a$ is $O$ (resp. $P$ ) if the length of the path (i.e. its number of edges) going from a root of $A$ to $a$ is even (resp. odd).

A move $a$ of $A$ is initial, denoted by $\vdash_{A} a$, if it is a root of $A$. If $b$ is a son of $a$ in $A$, we say that $a$ enables $b$, denoted by $a \vdash_{A} b$.

Definition 7 (Arrow)

Let $\left(A, \leq_{A}\right)$ and $\left(B, \leq_{B}\right)$ be two arenas, the arena $\left(A \rightarrow B, \leq_{A \rightarrow B}\right)$ is defined by:

- the set $A \rightarrow B$ is the disjoint union of $A$ and $B$;

- if $a \in A$ and $a^{\prime} \in A$ with $a \vdash_{A} a^{\prime}$, then $a \vdash_{A \rightarrow B} a^{\prime}$;

- if $b \in B$ and $b^{\prime} \in B$ with $b \vdash_{B} b^{\prime}$, then $b \vdash_{A \rightarrow B} b^{\prime}$;

- if $b \in B$ is a root and $a \in A$ is a root, then $b \vdash_{A \rightarrow B} a$.

\section{Definition 8 (Play)}

Let $A$ be an arena, a play $s$ on $A$ is a sequence of moves of alternated polarity of $A$ with, for each non initial move $b$ of $s$, a pointer to an earlier occurrence of move $a$ of $s$, called the justifier of $b$, such that $a \vdash_{A} b$.

The set of plays of $A$ is denoted by $\mathcal{P}_{A}$. We use the notation $t \leq{ }^{P} s$ if $t$ is a prefix of $s$ ending by a $P$-move. 
Definition 9 (View)

Let $A$ be an arena and $s$ be a play on $A$, the view $\ulcorner s\urcorner$ of $A$ is the sub-play of $s$ defined by:

- $\ulcorner s a\urcorner=\ulcorner s\urcorner a$ if $a$ is a $P$-move;

- $\ulcorner s a\urcorner=a$ if $a$ is an initial $O$-move;

- $\ulcorner s a t b\urcorner=\ulcorner s\urcorner a b$ is $b$ is an $O$-move justified by $a$.

\section{Definition 10 (Strategy)}

A strategy $\sigma$ on $A$, denoted by $\sigma: A$, is a non empty $P$-prefix closed set of even length plays of $A$ such that:

- determinism: if $s a b \in \sigma$ and $s a c \in \sigma$, then $s a b=s a c$.

- visibility: if $s a b \in \sigma$, the justifier of $b$ is in $\ulcorner s a\urcorner$.

- innocence: if $s a b \in \sigma, t \in \sigma, t a \in \mathcal{P}_{A}$ and $\ulcorner s a\urcorner=\ulcorner t a\urcorner$ then $t a b \in \sigma$.

Remark: Due to the innocence condition, a strategy is completely characterized by its views.

\section{Definition 11 (Composition)}

Let $A, B$ and $C$ be three arenas, an interaction sequence $u$ on $A, B$ and $C$ is a (possibly) non alternated play of $(A \rightarrow B) \rightarrow C$ such that $u \uparrow_{A \rightarrow B} \in \mathcal{P}_{A \rightarrow B}, u \uparrow_{B \rightarrow C} \in \mathcal{P}_{B \rightarrow C}$ and $u \uparrow_{A \rightarrow C} \in$ $\mathcal{P}_{A \rightarrow C}$. A move of $u$ in $A$ pointing to a move in $B$ is an initial move of $A$ and its justifier is an initial move of $B$, the play $u \uparrow_{A \rightarrow C}$ is obtained by choosing as a pointer for these initial moves of $A$ the justifier of their justifier which is an initial move of $C$. The set of the interaction sequences on $A, B$ and $C$ in denoted by $\operatorname{int}(A, B, C)$.

Let $\sigma: A \rightarrow B$ and $\tau: B \rightarrow C$ be two strategies, the composition of $\sigma$ and $\tau$ is the strategy $\sigma ; \tau=\left\{u\left\lceil_{A \rightarrow C} \mid u \in \operatorname{int}(A, B, C) \wedge u \uparrow_{A \rightarrow B} \in \sigma \wedge u \uparrow_{B \rightarrow C} \in \tau\right\}: A \rightarrow C\right.$.

\section{Lemma 3 (Zipping)}

Let $\sigma: A \rightarrow B$ and $\tau: B \rightarrow C$ be two strategies, if $s$ is a play in $\sigma ; \tau$, there exists at most one interaction sequence $u$ ending by a move in $A$ or $C$ such that $u \uparrow_{A}=s \uparrow_{A}$ and $u \Gamma_{C}=s \Gamma_{C}$.

Proof: Let $u$ and $v$ be two interaction sequences on $A, B$ and $C$ such that $u \uparrow_{A}=v \uparrow_{A}=s \Gamma_{A}$ and $u \uparrow_{C}=v \uparrow_{C}=s \uparrow_{C}$. We prove the result by induction on the length of $s$. If $s=\varepsilon$, we have $u=v=\varepsilon$. If $s=t m n$, let $u^{\prime}$ (resp. $v^{\prime}$ ) be the prefix of $u$ (resp. $v$ ) ending by the last move of $t$, by induction hypothesis, we have $u^{\prime}=v^{\prime}$. We decompose $u$ into $u=u^{\prime} m u^{\prime \prime} n$ and $v$ into $v=v^{\prime} m v^{\prime \prime} n$ so that $u^{\prime \prime}$ and $v^{\prime \prime}$ contain only moves in $B$. We must have $u^{\prime \prime}=v^{\prime \prime}$ since each Opponent move (resp. Player move) in these sub-sequences is uniquely determined by $\tau$ (resp. $\sigma$ ) which is a deterministic strategy.

\section{Definition 12 (Identity)}

Let $A$ be an arena, the identity strategy $i d_{A}$ is $i d_{A}=\left\{s \in \mathcal{P}_{A_{1} \rightarrow A_{2}} \mid \forall t \leq^{P} s, t\left\lceil A_{1}=t\left\lceil A_{2}\right\}: A \rightarrow A\right.\right.$ (the indexes are only used to distinguish occurrences).

\section{Lemma 4}

If $s$ is a sequence of moves of $A$ with pointers (possibly without alternation), there exists a unique play $s^{\prime} \in \mathcal{P}_{A_{1} \rightarrow A_{2}}$ of $i d_{A}$ such that $s^{\prime} \uparrow_{A_{1}}=s^{\prime} \uparrow_{A_{2}}=s$. 
Proof: By induction on the length of $s$. If $s=\varepsilon$, we have $s^{\prime}=\varepsilon$. If $s=t a$, by induction hypothesis, there exists a play $t^{\prime}$ of $i d_{A}$ such that $t^{\prime}\left\lceil A_{1}=t^{\prime}\left\lceil A_{2}=t\right.\right.$. If $a$ is an Opponent move, we have $s^{\prime}=t^{\prime} a_{2} a_{1}$ and if $a$ is a Player move, we have $s^{\prime}=t^{\prime} a_{1} a_{2}$ where $a_{i}$ is the copy of $a$ in $A_{i}$.

Definition 13 (Total strategy)

Let $\sigma: A$ be a strategy, $\sigma$ is total if whenever $s \in \sigma$ and $s a \in \mathcal{P}_{A}$, there exists some $b$ such that $s a b \in \sigma$.

\subsection{Isomorphisms}

We are going to characterize isomorphisms in the game model which is the key result of the paper.

\section{Proposition 2}

Let $f$ be a forest isomorphism from $A$ to $B,\left\{s \in \mathcal{P}_{A \rightarrow B} \mid \forall t \leq{ }^{P} s, f\left(t \uparrow_{A}\right)=t \uparrow_{B}\right\}$ is a strategy, called the strategy generated by $f$.

Proof: We denote this set of plays by $\sigma$, by definition $\sigma$ is a $P$-prefix closed set of even length plays of $A \rightarrow B$. We have to prove the three properties of strategies:

- If $s a b \in \sigma$ and $s a c \in \sigma$, we assume that $a$ is in $A$, this entails that $b$ and $c$ are in $B$, so that $s a b \uparrow_{B}=f\left(s a b \uparrow_{A}\right)=f\left(s a \uparrow_{A}\right)=f\left(s a c \uparrow_{A}\right)=s a c \uparrow_{B}$ and thus $s a b=s a c$.

- If $s a b \in \sigma$, we assume that $a$ is in $A$, this entails that $b$ is in $B$ and, since $s a b \uparrow_{B}=$ $f\left(s a b \uparrow_{A}\right)$, the justifier of $b$ is the move just before the justifier of $a$ in $s a b$ so that it is in the view $\ulcorner s a\urcorner$.

- If $s a b \in \sigma, t \in \sigma, t a \in \mathcal{P}_{A \rightarrow B}$ and $\ulcorner s a\urcorner=\ulcorner t a\urcorner$, we assume that $a$ is in $A$, this entails that $b$ is in $B$ and, we have $s a b \uparrow_{B}=f\left(s a b \uparrow_{A}\right)$ thus $b=f(a)$ and we conclude $t a b \uparrow_{B}=f\left(t a b \uparrow_{A}\right)$ that is $t a b \in \sigma$.

\section{Definition 14 (Game isomorphism)}

Let $A$ and $B$ be two negative arenas, a game isomorphism between $A$ and $B$ is a pair of strategies $\sigma: A \rightarrow B$ and $\tau: B \rightarrow A$ such that $\sigma ; \tau=i d_{A}$ and $\tau ; \sigma=i d_{B}$.

If there exists a game isomorphism between $A$ and $B$, we use the notation $A \simeq_{g} B$.

\section{Definition 15 (Zig-zag play)}

A play $s$ in $A \rightarrow B$ is zig-zag if:

- each $P$-move following an $O$-move in $A$ (resp. $B$ ) is in $B$ (resp. $A$ );

- each $P$-move in $A$ following an initial $O$-move in $B$ is justified by it;

- $s \uparrow_{A}$ and $s \uparrow_{B}$ have the same pointers.

If only the first two conditions are verified, $s$ is a pre-zig-zag play.

If $s$ is a pre-zig-zag play of even length in $A \rightarrow B$, we denote by $\bar{s}$ the unique pre-zig-zag play in $B \rightarrow A$ such that $\bar{s} \uparrow_{A}=s \uparrow_{A}$ and $\bar{s} \uparrow_{B}=s \uparrow_{B}$.

\section{Lemma 5 (Composition of zig-zag plays)}

If $s \in \mathcal{P}_{A \rightarrow B}$ is a pre-zig-zag play of even length, there exists an interaction sequence $\tilde{s} \in \operatorname{int}(B, A, B)$ such that $\tilde{s}\left\lceil_{B \rightarrow A}=\bar{s}\right.$ and $\tilde{s} \uparrow_{A \rightarrow B}=s$. 
Proof: We prove the result by induction on the length of $s$. If $s=\varepsilon$, we have $\bar{s}=\varepsilon$ and $\tilde{s}=\varepsilon$. If $s=t a b$, by induction hypothesis, there exists an interaction sequence $\tilde{t}$ such that $\tilde{t} \uparrow_{B \rightarrow A}=\bar{t}$ and $\tilde{t} \uparrow_{A \rightarrow B}=t$. If $a$ is in $A$ thus $b$ is in $B$ (the case $a$ in $B$ is the same), we consider $\tilde{s}=\tilde{t} b_{1} a b_{2}$ where $b_{1}$ is the copy of $b$ in the leftmost $B$ and $b_{2}$ is the copy of $b$ in the rightmost $B$.

\section{Theorem 1 (Strict isomorphisms)}

If there exists a game isomorphism $(\sigma, \tau)$ between $A$ and $B$, then there exists a forest isomorphism $f$ from $A$ to $B$. Moreover $\sigma$ (resp. $\tau$ ) is the strategy generated by $f$ (resp. $f^{-1}$ ).

Proof: We first show by induction on the even number $k$ that if $s \in \sigma$ with length $k$ then $s$ is a zig-zag play and $\{s|s \in \tau \wedge| s \mid=k\}=\{\bar{s}|s \in \sigma \wedge| s \mid=k\}$ :

- If $k=0$ then $s=\varepsilon$ and the result is immediate.

- If $k=k^{\prime}+2$ and $s$ is a play in $\sigma$ of length $k$, we have $s=t m n$ with $t \in \sigma$ and by induction hypothesis $t$ is zig-zag and $\bar{t} \in \tau$. We assume $m \in B$ (the case $m \in A$ is similar), if $n \in B$ then, using lemma $5, \tilde{t} m n \in \operatorname{int}(B, A, B)$ is such that $\tilde{t} m n \uparrow_{B \rightarrow A}=\tilde{t} \uparrow_{B \rightarrow A}=\bar{s} \in \tau$ and $\tilde{t} m n \uparrow_{A \rightarrow B}=\tilde{t} \uparrow_{A \rightarrow B} m n=t m n \in \sigma$, so that $\tilde{t} m n \uparrow_{B \rightarrow B} \in \tau ; \sigma=i d_{B}$ but this is impossible because a play in $i d_{B}$ cannot contain two successive moves in the same $B$. This entails $n \in A$.

According to lemma 4 , we consider the play $s^{\prime} \in \mathcal{P}_{B_{1} \rightarrow B_{2}}$ of $i d_{B}$ such that $s^{\prime} \uparrow_{B_{1}}=$ $s^{\prime} \uparrow_{B_{2}}=s \uparrow_{B}=t \uparrow_{B} m$. By definition of the composition $\tau ; \sigma$, there exists $u \in \operatorname{int}(B, A, B)$ such that $u \uparrow_{B \rightarrow B}=s^{\prime}, u \uparrow_{B \rightarrow A} \in \tau$ and $u \uparrow_{A \rightarrow B} \in \sigma$. By lemma $3, u=\tilde{t} m n m$ which entails that $n$ is justified by $m$ in $s$ if $m$ is initial (otherwise $u \uparrow_{B \rightarrow B} \notin i d_{B}$ ) so that $s$ is a pre-zig-zag play. Moreover $\tilde{t} m n m \uparrow_{B \rightarrow A}=\tilde{s} \uparrow_{B \rightarrow A}=\bar{s} \in \tau$ so that $\{\bar{s}|s \in \sigma \wedge| s \mid=k\} \subset\{s|s \in \tau \wedge| s \mid=k\}$. We obtain the converse in the same way.

We still have to show that $s$ is a zig-zag play. The justifier of $n$ in $s \uparrow_{A}$ must be a move before the justifier of $m$ in $s \uparrow_{B}$ by visibility of $\sigma$, and it must be the move just before it, otherwise the justifier of $m$ in $\bar{t} n m \in \tau$ is not in $\ulcorner\bar{t} n\urcorner$, contradicting the visibility condition for $\tau$.

We have shown that $\tau=\{\bar{s} \mid s \in \sigma\}$. We have almost shown that $\sigma$ is total: if $s \in \sigma$ and $s m \in \mathcal{P}_{A \rightarrow B}$, we assume $m \in B$ (the case $m \in A$ is similar), we consider the play $s^{\prime} \in \mathcal{P}_{B_{1} \rightarrow B_{2}}$ of $i d_{B}$ such that $s^{\prime} \uparrow_{B_{1}}=s^{\prime} \uparrow_{B_{2}}=s m \uparrow_{B}$. By definition of the composition $\tau ; \sigma$, there exists $u \in \operatorname{int}(B, A, B)$ such that $u \uparrow_{B \rightarrow B}=s^{\prime}, u \uparrow_{B \rightarrow A} \in \tau$ and $u \uparrow_{A \rightarrow B} \in \sigma$. We can write $u=u^{\prime} m n m$ for some $n$ since $\sigma$ and $\tau$ contain only zig-zag plays, and by lemma 3 , we have $u^{\prime}=\tilde{s}$ so that $\left.\tilde{s} m n m\right|_{A \rightarrow B}=s m n \in \sigma$. This immediately gives $\tau$ also total.

Let $a$ be a move of $A$ and $a_{1} \ldots a_{p}$ be the sequence of moves of $A$ such that $a_{1}$ is initial, $a_{i}$ enables $a_{i+1}$ for each $1 \leq i \leq p-1$ and $a_{p}=a$, we are going to define a function $f$ from the moves of $A$ to the moves of $B$ by induction on $p$. In fact we enrich the induction hypothesis by requiring that $f\left(a_{0}\right) a_{0} a_{1} f\left(a_{1}\right) f\left(a_{2}\right) a_{2} \ldots \in \sigma$. If $p=1$, we look at the unique play of the shape $a b$ in $\tau$ (which exists by totality) and we define $f(a)=b$. If $p=p^{\prime}+1$ with $p$ odd, we have by induction hypothesis $a_{1} f\left(a_{1}\right) f\left(a_{2}\right) a_{2} a_{3} f\left(a_{3}\right) \ldots f\left(a_{p^{\prime}}\right) a_{p^{\prime}} \in \tau$, let $f\left(a_{p}\right)$ be the unique move such that $a_{1} f\left(a_{1}\right) f\left(a_{2}\right) a_{2} a_{3} f\left(a_{3}\right) \ldots f\left(a_{p^{\prime}}\right) a_{p^{\prime}} a_{p} f\left(a_{p}\right) \in \tau$ which exists by totality of $\tau$. If $p=p^{\prime}+1$ with $p$ even, we have by induction hypothesis $f\left(a_{1}\right) a_{1} a_{2} f\left(a_{2}\right) f\left(a_{3}\right) a_{3} \ldots f\left(a_{p^{\prime}}\right) a_{p^{\prime}} \in$ $\sigma$, let $f\left(a_{p}\right)$ be the unique move such that $f\left(a_{1}\right) a_{1} a_{2} f\left(a_{2}\right) f\left(a_{3}\right) a_{3} \ldots f\left(a_{p^{\prime}}\right) a_{p^{\prime}} a_{p} f\left(a_{p}\right) \in \sigma$ which exists by totality of $\sigma$.

In the same way, we can associate a function $g$ with $\tau$ and we easily verify that $f \circ g$ is the identity on the moves of $B$ and $g \circ f$ is the identity on the moves of $A$ so that $f$ is a 
bijection. Moreover, by construction, if $a \leq a^{\prime}$ in $A$ we have $f(a) \leq f\left(a^{\prime}\right)$ in $B$, so that $f$ is an isomorphism between the forests $A$ and $B$.

Finally, we want to show that if $\sigma$ is innocent, $\sigma$ is the strategy generated by $f$. Let $\sigma_{f}$ be this strategy, we just have to show that if $s$ is a $P$-view then $s \in \sigma \Longleftrightarrow s \in \sigma_{f}$. Let $s$ be a zig-zag $P$-view in $A \rightarrow B$, we prove by induction on the length of $s$ that $s \uparrow_{A}$ is a sequence $a_{1} \ldots a_{p}$ such that $a_{i}$ justifies $a_{i+1}$ for $1 \leq i \leq p-1$ and $s \uparrow_{B}$ is a sequence $b_{1} \ldots b_{q}$ such that $b_{j}$ justifies $b_{j+1}$ for $1 \leq j \leq q-1$. If $s=\varepsilon$ the result is straightforward. If $s=m n, m \in B$ and $n \in A$ are initial. If $s=t n^{\prime} m^{\prime} m n, m$ is justified by $m^{\prime}$ since $s$ is a $P$-view and $n$ is justified by $n^{\prime}$ since $s$ is zig-zag. We can then show, by an easy induction on the length of $s$, that $s \in \sigma \Longleftrightarrow s \in \sigma_{f}$.

Remark: In the setting of sequential algorithms [7], Berry and Curien have given a similar characterization of isomorphisms based on a decomposition of algorithms into a purely functional part (giving the underlying order-theoretic isomorphism) and an index choice part.

\section{The game model of LLP}

In order to characterize the isomorphisms of types in LLP, we give the game interpretation of LLP and we show that it gives a denotational model. In fact the relation between LLP and its game model is very much stronger (completeness, ...) but we just need soundness here. A precise comparison between LLP and the same game model is given in [16].

\section{Definition 16 (Polarized arena)}

A polarized arena $A$ is an arena with a polarity $\pi_{A}$ which is $P$ or $O$ (also denoted by + or - ).

The polarity $\lambda_{A}(a)$ of a move $a$ is $\pi_{A}$ (resp. $\overline{\pi_{A}}$ ) if the length of the path going from a root of $A$ to $a$ is even (resp. odd).

\section{Definition 17 (Constructions of arenas)}

We consider the following constructions on polarized arenas:

Dual. If $A$ is an arena, its dual $A^{\perp}$ is obtained by changing its polarity.

Empty. There are two empty polarized arenas: the positive one and the negative one.

Unit. The unit arenas are the forests reduced to one node $\circ$ and with polarity $O$ or $P$.

Sum. If $A$ and $B$ are two arenas of the same polarity, $A+B$ is the disjoint union of the two forests.

Product. If $A$ and $B$ are two arenas of the same polarity, the trees of $A \times B$ are obtained by taking a tree in $A$ and a tree in $B$ and by identifying their roots. More formally, if $A^{r}$ (resp. $B^{r}$ ) are the roots of $A$ (resp. $B$ ):

- the underlying set of $A \times B$ is $\left(A^{r} \times B^{r}\right)+\left(A \backslash A^{r}\right)+\left(B \backslash B^{r}\right)$;

- if $a \in A^{r}, a^{\prime} \in A \backslash A^{r}, b \in B^{r}$ and $a \vdash_{A} a^{\prime}$ then $(a, b) \vdash_{A \times B} a^{\prime}$;

- if $a \in A^{r}, b \in B^{r}, b^{\prime} \in B \backslash B^{r}$ and $b \vdash_{B} b^{\prime}$ then $(a, b) \vdash_{A \times B} b^{\prime}$;

- if $a \in A \backslash A^{r}, a^{\prime} \in A \backslash A^{r}$ and $a \vdash_{A} a^{\prime}$ then $a \vdash_{A \times B} a^{\prime}$;

- if $b \in B \backslash B^{r}, b^{\prime} \in B \backslash B^{r}$ and $b \vdash_{B} b^{\prime}$ then $b \vdash_{A \times B} b^{\prime}$;

- $\pi_{A \times B}=\pi_{A}=\pi_{B}$. 
Lift. If $A$ is an arena of polarity $\pi_{A}, \uparrow A$ is obtained by adding a unique new root $\circ$ under all the trees of $A$. It is an arena of polarity $\overline{\pi_{A}}$.

Remark: If $N$ and $M$ are two negative arenas, $N \rightarrow M \simeq_{f} \uparrow N^{\perp} \times M$, and this corresponds to an encoding of implication in Linear Logic: $A \rightarrow B=$ ? $A^{\perp}$ भ8 $B$.

A polarized formula $A$ is interpreted by a polarized arena $A^{\star}$ of the same polarity. Given such an interpretation for the variables, we then have:

$$
\begin{array}{rlrlrl}
0^{\star} & =(\emptyset, P) & T^{\star} & = & (\emptyset, O) \\
1^{\star} & =(\circ, P) & \perp^{\star} & = & (\circ, O) \\
(P \oplus Q)^{\star} & = & P^{\star}+Q^{\star} & (N \& M)^{\star} & = & N^{\star}+M^{\star} \\
(P \otimes Q)^{\star} & = & P^{\star} \times Q^{\star} & (N>M)^{\star} & = & N^{\star} \times M^{\star} \\
(! N)^{\star} & = & \uparrow N^{\star} & (? P)^{\star} & = & \uparrow P^{\star}
\end{array}
$$

with the property $A^{\star \perp}=A^{\perp^{\star}}$.

\section{Example 1}

The polarized arena associated with ? $(1 \oplus !(? ! \top \& \perp)) \&(?(! \perp \oplus 1)$ \& $? !(\perp \& \perp))$ is:

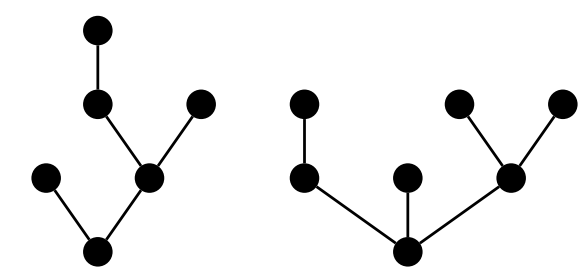

with polarity $O$.

\section{Definition 18 (Central strategy)}

Let $\sigma: A \rightarrow B$ be a strategy,

- $\sigma$ is linear if in each play of $\sigma$, each initial move in $B$ has exactly one move in $A$ justified by it.

- $\sigma$ is strict if, for each initial move $b$ in $B$, it contains a play $b a$ with $a$ in $A$.

- $\sigma$ is central, denoted by $\sigma: A \dot{\rightarrow}$, if it is strict and linear.

\section{Definition 19 (Product)}

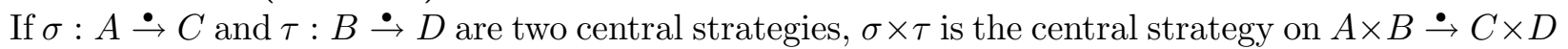
defined by:

$$
\sigma \times \tau=\left\{s \in \mathcal{P}_{A \times B \rightarrow C \times D} \mid s \uparrow_{A \rightarrow C} \in \sigma \wedge s \uparrow_{B \rightarrow D} \in \tau\right\}
$$

\section{Definition 20 (Weakening)}

The views of the weakening strategy $w_{N}$ are $\varepsilon$ and $\{n \circ \mid n$ initial in $N\}$ which give a central strategy on $\perp \stackrel{\bullet}{\rightarrow}$.

\section{Definition 21 (Contraction)}

If $s$ is a play on $N_{1} \times N_{2} \rightarrow N_{0}$ we define $s_{i}(i=1,2)$ to be the sub-sequence of $s$ containing the initial moves in $N_{0}$, the moves in $N_{i}$ and the moves in $N_{0}$ such that the last move before in $N_{1} \times N_{2}$ is in $N_{i}$.

The contraction strategy is $c_{N}=\left\{s \in \mathcal{P}_{N_{1} \times N_{2} \rightarrow N_{0}} \mid \forall t \leq{ }^{P} s, t_{1} \in i d_{N} \wedge t_{2} \in i d_{N}\right\}: N \times N \dot{\rightarrow}$. 
By lemma 1, a provable sequent of LLP is $\vdash \mathcal{N}$, $\Pi$ where $\Pi$ is empty or is a positive formula. The interpretation of a proof $\pi$ of $\vdash \mathcal{N}, \Pi$ is a strategy $\pi^{\star}$ on $\Pi^{\perp} \doteq \mathcal{N}$ (this is a notation for $\mathcal{N}^{\star}$ if $\Pi$ is empty and $P^{\star \perp} \dot{\bullet} \mathcal{N}^{\star}$ if $\Pi=P$ ) central if $\Pi$ is not empty.

The strategy $\pi^{\star}$ is defined by induction on $\pi$ :

(ax) $a x^{\star}=i d_{N}$.

(cut) if $\Gamma=\Gamma^{\prime}, \Pi, \pi_{1}^{\star}: \Pi^{\perp} \dot{\rightarrow} \Gamma^{\prime}$ ช $N$ and $\pi_{2}^{\star}: N \stackrel{\bullet}{\rightarrow}$, the strategy $\pi^{\star}$ is $\pi_{1}^{\star} ;\left(i d_{\Gamma^{\prime}} \times \pi_{2}^{\star}\right): \Pi^{\perp} \dot{\rightarrow}$ $\Gamma^{\prime} 28 \Delta$.

(2) this rule doesn't modify the interpretation.

$(\otimes)$ if $\pi_{1}^{\star}: P^{\perp} \dot{\rightarrow} \Gamma$ and $\pi_{2}^{\star}: Q^{\perp} \dot{\rightarrow} \Delta$, the strategy $\pi^{\star}$ is $\pi_{1}^{\star} \times \pi_{2}^{\star}:(P \otimes Q)^{\perp} \dot{\bullet} \Gamma \not 8 \Delta$.

(\&) if $\Gamma=\Gamma^{\prime}, \Pi, \pi_{1}^{\star}: \Pi^{\perp} \dot{\hookrightarrow} \Gamma^{\prime} \not \gamma N$ and $\pi_{2}^{\star}: \Pi^{\perp} \dot{\bullet} \Gamma^{\prime} \not \gamma M$, the views of $\pi^{\star}$ are the views of $\pi_{1}^{\star}$ and the views of $\pi_{2}^{\star}$ which give a strategy on $\Pi^{\perp} \dot{\bullet} \Gamma^{\prime}$ \&8 $(M \& N)$.

$\left(\oplus_{i}\right)$ if $\pi_{1}^{\star}: P_{i}^{\perp} \dot{\rightarrow} \Gamma, \pi^{\star}$ is $\pi_{1}^{\star}$ seen as a strategy on $\left(P_{1} \oplus P_{2}\right)^{\perp} \dot{\hookrightarrow} \Gamma$.

(!) if $\pi_{1}^{\star}: \mathcal{N} \not \gamma N$, a view of $\pi^{\star}:(! N)^{\perp} \dot{\rightarrow} \mathcal{N}$ is $\varepsilon$, or $m \circ$ with $m$ initial in $\mathcal{N}$, or mons where $(m, n) s$ is a view of $\pi_{1}^{\star}$.

$(? d)$ if $\pi_{1}^{\star}: P^{\perp} \dot{\rightarrow} \Gamma$, we have $\pi^{\star}=\{\varepsilon\} \cup\left\{s[(a, \circ) / a] \mid s \in \pi_{1}^{\star}\right\}: \Gamma$ P $? P$ where $s\left[{ }^{(a, \circ)} / a\right]$ is obtained from $s$ by replacing any initial move $a$ by $(a, \circ)$.

$(? w)$ if $\Gamma=\Gamma^{\prime}, \Pi$ and $\pi_{1}^{\star}: \Pi^{\perp} \dot{\bullet} \Gamma^{\prime}$, we can define a strategy $\sigma$ on $\Pi^{\perp} \dot{\rightarrow} \Gamma^{\prime} \not \gamma \perp$ as in the case of a $\perp$-rule, we have $\pi^{\star}=\sigma ;\left(i d_{\Gamma^{\prime}} \times w_{N}\right): \Pi^{\perp} \dot{\hookrightarrow} \Gamma^{\prime} \not \gamma N$.

$(? c)$ if $\Gamma=\Gamma^{\prime}, \Pi$ and $\pi_{1}^{\star}: \Pi^{\perp} \dot{\rightarrow} \Gamma^{\prime} \not \gamma N$ ช $N$, we have $\pi^{\star}=\pi_{1}^{\star} ;\left(i d_{\Gamma^{\prime}} \times c_{N}\right): \Pi^{\perp} \dot{\rightarrow} \Gamma^{\prime} \not \gamma N$.

( $丁)$ if $\Gamma=\Gamma^{\prime}, \Pi$, we have $\top^{\star}=\{\varepsilon\}: \Pi^{\perp} \dot{\rightarrow} \Gamma^{\prime} \not \supset \top$.

$(\perp)$ if $\Gamma=\Gamma^{\prime}, \Pi$ and $\pi_{1}^{\star}: \Pi^{\perp} \dot{\rightarrow} \Gamma^{\prime}$, we have $\pi^{\star}=\{\varepsilon\} \cup\left\{s\left[{ }^{(a, o)} /{ }_{a}\right] \mid s \in \pi_{1}^{\star}\right\}: \Pi^{\perp} \dot{\rightarrow} \Gamma^{\prime} \not \perp \perp$.

(1) The views of $1^{\star}$ are $\varepsilon$ and $\circ_{\perp} \circ_{1}$ which give a strategy on $1^{\perp} \dot{\rightarrow} \perp$.

Proposition 3 (Soundness)

If $\pi$ and $\pi^{\prime}$ are two proofs of $\vdash \Gamma$ in LLP, $\pi={ }_{\beta \eta} \pi^{\prime}$ entails $\pi^{\star}=\pi^{\prime \star}$.

Proof: The proof of this result is given in [16].

\section{$5 \quad$ Polarized type isomorphisms}

Using the two previous sections, we can move from the study of isomorphisms in LLP to the study of isomorphic forests: $A \simeq \mathrm{LLP} B \Rightarrow A^{\star} \simeq_{g} B^{\star} \Rightarrow A^{\star} \simeq_{f} B^{\star}$.

\subsection{Polarized isomorphisms without variables}

The game interpretation of variables is not precise enough to directly obtain a characterization of types isomorphisms with variables. This is why we first study the variable free case.

\section{Lemma 6 (Additive translation)}

Let $A$ be a polarized formula without variable, there exists a formula $A^{a}$ containing only the connectives $\top, 0, \&, \oplus, !$ and ?, such that $A \simeq_{\mathcal{E}} A^{a}$. 
PROOF: We consider the following rewriting system (up to commutativity of the binary connectives) which is derived from the theory $\mathcal{E}$ :

$$
\begin{aligned}
& N \gamma \perp \rightsquigarrow N \\
& N \& \top \rightsquigarrow N \\
& N \not \supset \top \rightsquigarrow \top \\
& N \not 8(M \& L) \rightsquigarrow(N \not 8 M) \&(N \not 2 L) \\
& ? P \not P ? Q \rightsquigarrow ?(P \oplus Q) \\
& \perp \rightsquigarrow ? 0
\end{aligned}
$$

$$
\begin{aligned}
P \otimes 1 & \rightsquigarrow P \\
P \oplus 0 & \rightsquigarrow P \\
P \otimes 0 & \rightsquigarrow 0 \\
P \otimes(Q \oplus R) & \rightsquigarrow(P \otimes Q) \oplus(P \otimes R) \\
! N \otimes ! M & \rightsquigarrow !(N \& M) \\
1 & \rightsquigarrow ! \top
\end{aligned}
$$

To show the termination of this system, we define a function $\varphi$ which associates with each polarized formula $A$ without variable an integer $\varphi(A) \geq 2$ by:

$$
\begin{array}{ccccc}
\varphi(A>B) & = & \varphi(A \otimes B) & = & \varphi(A) \varphi(B) \\
\varphi(A \& B) & = & \varphi(A \oplus B) & = & \varphi(A)+\varphi(B)+1 \\
\varphi(! A) & = & \varphi(? A) & = & \varphi(A)+1 \\
\varphi(\perp) & = & \varphi(1) & = & 4 \\
\varphi(\top) & = & \varphi(0) & = & 2
\end{array}
$$

For any rewriting rule $A \rightsquigarrow B$, we have $\varphi(A)>\varphi(B)$.

We have to show that a normal form doesn't contain any $\not 2, \otimes, \perp$ and 1 . By $\perp \rightsquigarrow ? 0$ and $1 \rightsquigarrow ! T$, the case of $\perp$ and 1 is immediate. We consider the case of $\otimes$ ( $\not \partial$ is the same), if $A \otimes B$ appears in the formula with $A$ and $B$ without $28, \otimes, \perp$ and 1 , the main connective of $A$ and $B$ must be $\oplus, 0$ or !. If one of these two main connectives is $\oplus$ or 0 , we can apply a rule $P \otimes(Q \oplus R) \rightsquigarrow(P \otimes Q) \oplus(P \otimes R)$ or $P \otimes 0 \rightsquigarrow 0$ and if both of them are !, we can apply $! N \otimes ! M \rightsquigarrow !(N \& M)$.

\section{Lemma 7 (Additive arenas)}

Let $A$ and $B$ be two polarized formulas without variable containing only the connectives $\top, 0, \&$, $\oplus$, ! and ?, and such that $A^{\star} \simeq_{f} B^{\star}$, we have $A \simeq_{\mathcal{E}} B$.

Proof: By induction on the size of $A^{\star}$, with $A$ and $B$ positive:

- If $A^{\star}$ is empty, $B^{\star}$ is also empty and $A=B=0$.

- If $A^{\star}$ is a tree, $B^{\star}$ is also a tree and $A \simeq_{\mathcal{E}} ! A^{\prime}$ and $B \simeq_{\mathcal{E}} ! B^{\prime}$ (we need $\simeq_{\mathcal{E}}$ in the case $A=! A^{\prime} \oplus 0$ for example). The forest $A^{\prime \star}$ (resp. $B^{\prime \star}$ ) is obtained by removing the root of $A^{\star}\left(\right.$ resp. $\left.B^{\star}\right)$ so that $A^{\prime \star} \simeq_{f} B^{\prime \star}$ and by induction hypothesis, $A^{\prime} \simeq_{\mathcal{E}} B^{\prime}$ and finally $A \simeq_{\mathcal{E}} B$.

- If $A^{\star}$ contains $k \geq 2$ trees, $B^{\star}$ also contains $k$ trees and $A$ is obtained from $k$ formulas $A_{1}, \ldots, A_{k}$ by adding the correct $\oplus$ connectives, this entails by associativity: $A \simeq_{\mathcal{E}}$ $\left(\left(A_{1} \oplus A_{2}\right) \cdots \oplus A_{k-1}\right) \oplus A_{k}$ where each $A_{i}^{\star}$ is one of the trees of $A^{\star}$. In the same way, we get $B \simeq_{\mathcal{E}}\left(\left(B_{1} \oplus B_{2}\right) \cdots \oplus B_{k-1}\right) \oplus B_{k}$ and each $B_{i}^{\star}$ is one of the trees of $B^{\star}$. From $A^{\star} \simeq_{f} B^{\star}$, we can find a permutation $\theta$ of the trees of $A^{\star}$ such that for each $1 \leq i \leq k$, $A_{\theta(i)}^{\star} \simeq_{f} B_{i}^{\star}$. By induction hypothesis, $A_{\theta(i)} \simeq_{\mathcal{E}} B_{i}$ so that using commutativity of $\oplus$ in $\mathcal{E}$ we have $A \simeq_{\mathcal{E}} B$.

Proposition 4 (Variable free polarized isomorphisms)

Let $A$ and $B$ be two polarized formulas without variable,

$$
A \simeq_{\mathrm{LLP}} B \Longleftrightarrow A^{\star} \simeq_{g} B^{\star} \Longleftrightarrow A^{\star} \simeq_{f} B^{\star} \Longleftrightarrow A \simeq_{\mathcal{E}} B
$$


Proof: We prove the following implications:

- $A \simeq_{\mathcal{E}} B \Rightarrow A \simeq_{\mathrm{LLP}} B$, by lemma 2 .

- $A \simeq \mathrm{LLP} B \Rightarrow A^{\star} \simeq{ }_{g} B^{\star}$, by soundness of the game model (proposition 3).

- $A^{\star} \simeq_{g} B^{\star} \Rightarrow A^{\star} \simeq_{f} B^{\star}$, by theorem 1 .

- $A^{\star} \simeq_{f} B^{\star} \Rightarrow A \simeq_{\mathcal{E}} B$, we have $A \simeq_{\mathcal{E}} A^{a}$ and $B \simeq_{\mathcal{E}} B^{a}$ by lemma 6 , and this entails with the previous implications: $A^{a \star} \simeq_{f} A^{\star} \simeq_{f} B^{\star} \simeq_{f} B^{a \star}$. By lemma 7 we can deduce $A^{a} \simeq_{\mathcal{E}} B^{a}$ and finally $A \simeq_{\mathcal{E}} B$.

\subsection{Recovering variables}

The last result allows to forget everything about games. We show that the correspondence between (isomorphic) polarized formulas and forests can be extended with variables.

\section{Lemma 8 (Quasi-multiplicative translation)}

Let $A$ be a polarized formula (possibly with variables), there exists a formula $A^{m}$ containing none of the connectives $T, 0, \&, \oplus$ except in head position, and such that $A \simeq_{\mathcal{E}} A^{m}$.

Proof: We use the same proof as for lemma 6 but we reverse the following rewriting rules:

$$
\begin{aligned}
& ?(P \oplus Q) \rightsquigarrow ? P \not \gamma ? Q \quad !(N \& M) \rightsquigarrow \quad ! N \otimes ! M \\
& ? 0 \rightsquigarrow \perp \quad \text { ! } \rightsquigarrow 1
\end{aligned}
$$

and we use:

$$
\begin{array}{ccccc}
\varphi(A \ngtr B) & = & \varphi(A \otimes B) & = & \varphi(A) \varphi(B) \\
\varphi(A \& B) & = & \varphi(A \oplus B) & = & \varphi(A)+\varphi(B)+1 \\
\varphi(! A) & = & \varphi(? A) & = & \varphi(A) ! \\
\varphi(\perp) & = & \varphi(1) & = & 2 \\
\varphi(\top) & = & \varphi(0) & = & 3 \\
\varphi\left(X^{\perp}\right) & = & \varphi(X) & = & 2
\end{array}
$$

We have to show that a normal form doesn't contain any $\top, 0, \&, \oplus$ except in head position. If there is such a connective in a normal form which is not in head position, we consider one such connective with a different connective just above it. We first consider the case of 0 ( $T$ is the same), if the connective above it is a $\otimes$ we can apply $A \otimes 0 \rightsquigarrow 0$, if it is a $\oplus$ we can apply $A \oplus 0 \rightsquigarrow A$ and if it is a ? we can apply ?0 $\rightsquigarrow \perp$. For a $\oplus$ connective (\& is the same), if the connective above it is a $\otimes$ we can apply $A \otimes(B \oplus C) \rightsquigarrow(A \otimes B) \oplus(A \otimes C)$ and if it is a ? we can apply ? $(A \oplus B) \rightsquigarrow ? A$ ช ? B.

\section{Definition 22 (Atomized forests)}

An atomized forest is a forest with nodes labeled by finite multi-sets (possibly empty) of positive variables.

We can generalize the interpretation of LLP formulas by forests to an interpretation of LLP formulas by atomized forests, by associating to the variable $X$ (resp. $X^{\perp}$ ) the one point positive (resp. negative) forest labeled by $[X]$. The product of atomized forests is obtained with a union of the labeling multi-sets for the identified roots.

\section{Example 2}

The interpretation of $?\left(X^{\perp} \otimes Y^{\perp} \otimes ! 1\right) \& ?\left(Z^{\perp} \otimes(! X \oplus 1)\right)$ is the negative atomized forest: 


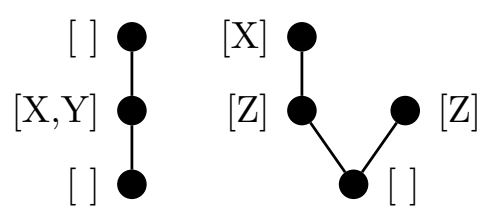

\section{Definition 23 (Atomized forest morphism)}

Let $A$ and $B$ be two atomized forests, an atomized forest morphism from $A$ to $B$ is a forest morphism $f$ from $A$ to $B$ such that, if $n$ is a node of $A$, the multi-set of variables of $n$ is included in the multi-set of $f(n)$.

\section{Definition 24 (Atomized forest substitution)}

A forest substitution $\theta$ is a partial function from positive variables to atomized forests.

If $\theta$ is a forest substitution and $A$ is a forest, the forest $A \theta$ is obtained by replacing, for each $X$ such that $\theta(X)$ is defined, every node $n$ of $A$ which contains $k$ occurrences of $X$ in its associated multi-set by the forest $\theta(X) \times \cdots \times \theta(X), k$ times (this preserves the existing sons of $n$ except if $\theta(X)$ is empty).

\section{Example 3}

The application of the substitution $Y \mapsto \emptyset$ and $Z \mapsto \complement^{[W]}$ [ to the forest of example 2 leads to:

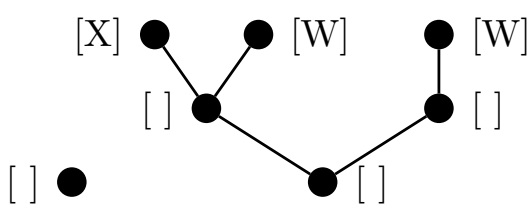

\section{Lemma 9}

If $A$ is a polarized formula and $\theta$ is a substitution, we can associate with $\theta$ a forest substitution $\theta^{\star}$ by $\theta^{\star}(X)=(\theta(X))^{\star}$ for any $X$. We have $(A \theta)^{\star}=A^{\star} \theta^{\star}$.

\section{Lemma 10 (Quasi-multiplicative arenas)}

Let $A$ and $B$ be two polarized formulas containing none of the connectives $\top, 0$, \& and $\oplus$ except in head position, and such that $A^{\star} \simeq_{f} B^{\star}$, we have $A \simeq_{\mathcal{E}} B$.

Proof: By induction on the size of $A^{\star}$, with $A$ and $B$ positive.

- If $A^{\star}$ and $B^{\star}$ are empty, $A=B=0$.

- If $A^{\star}$ and $B^{\star}$ are two trees, let $k$ be the number of sons of its root (which is the same for $A^{\star}$ and $B^{\star}$ ) and $A_{1}^{\star}, \ldots, A_{k}^{\star}\left(\right.$ resp. $B_{1}^{\star}, \ldots, B_{k}^{\star}$ ) be the immediate sub-trees of $A^{\star}$ (resp. $\left.B^{\star}\right)$. From $A^{\star} \simeq_{f} B^{\star}$ we can deduce a permutation $\theta$ of the $A_{i}^{\star}$ 's such that $A_{\theta(i)}^{\star} \simeq_{f} B_{i}^{\star}$. By induction hypothesis, we can find $k$ formulas $A_{1}, \ldots, A_{k}$ and $k$ formulas $B_{1}, \ldots, B_{k}$ such that, up to associativity $A \simeq_{\mathcal{E}} A_{1} \otimes \cdots \otimes A_{k}$ and $B \simeq_{\mathcal{E}} B_{1} \otimes \cdots \otimes B_{k}$, that is, up to commutativity, $A \simeq_{\mathcal{E}} B$.

- If $A^{\star}$ and $B^{\star}$ are two forests containing $k \geq 2$ trees, let $A_{1}^{\star}, \ldots, A_{k}^{\star}$ (resp. $B_{1}^{\star}, \ldots, B_{k}^{\star}$ ) be these trees, we have by induction hypothesis $A_{i} \simeq_{\mathcal{E}} B_{i}$ and thus $A \simeq_{\mathcal{E}} A_{1} \oplus \cdots \oplus A_{k} \simeq_{\mathcal{E}}$ $B_{1} \oplus \cdots \oplus B_{k} \simeq_{\mathcal{E}} B$.

Remark: It would also be possible to use an adaptation of the additive translation in the previous lemma but the quasi-multiplicative one makes things simpler. 
The main result of the paper follows, it gives two characterizations of polarized isomorphisms: a "geometrical" one (two types are isomorphic if the associated forests are isomorphic) and an equational one.

\section{Theorem 2 (Polarized isomorphisms)}

Let $A$ and $B$ be two polarized formulas,

$$
A \simeq \mathrm{LLP} B \Longleftrightarrow A^{\star} \simeq_{f} B^{\star} \Longleftrightarrow A \simeq_{\mathcal{E}} B
$$

ProOF: We prove the following implications:

- $A \simeq_{\mathcal{E}} B \Rightarrow A \simeq_{\mathrm{LLP}} B$, by lemma 2 .

- $A \simeq_{\mathrm{LLP}} B \Rightarrow A^{\star} \simeq_{f} B^{\star}$, for any substitution $\theta$ we have $A \theta \simeq_{\mathrm{LLP}} B \theta$. In particular if we consider the set of variables indexed by natural numbers: $X_{1}, X_{2}, \ldots$ and if $h$ is the height of $A^{\star}$, we can define the substitution $\theta_{h}$ given by $X_{i} \mapsto ! ? ! \cdots ? 1$ or $! ? ! \cdots ! \perp$ with exactly $i(h+1)-1$ exponential connectives. We have $A \theta_{h} \simeq \mathrm{LLP} B \theta_{h}$ and $A \theta_{h}$ and $B \theta_{h}$ are closed formulas, so that by proposition 4 we have $\left(A \theta_{h}\right)^{\star} \simeq_{f}\left(B \theta_{h}\right)^{\star}$ and by lemma 9 $A^{\star} \theta_{h}^{\star} \simeq_{f} B^{\star} \theta_{h}^{\star}$. We still have to deduce $A^{\star} \simeq_{f} B^{\star}$.

The forest $A^{\star} \theta_{h}^{\star}$ is $A^{\star}\left[{ }^{c_{i}} / X_{i}\right]$ where $c_{i}$ is the branch of length $i(h+1)$. We prove the result by induction on the number of elements of all the multi-sets associated with the nodes of $A^{\star}$. If all these multi-sets are empty, we have $A^{\star} \theta_{h}^{\star}=A^{\star}$ and we can conclude. If there are $k+1$ occurrences of variables, let $n$ be a node of $A^{\star} \theta_{h}^{\star}$ of maximal height, its height is of the shape $k+i_{0}(h+1)$ with $0 \leq k \leq h$. Let $m$ be the node which is $i_{0}(h+1)$ levels under $n$, this node has an occurrence $X$ of $X_{i_{0}}$ associated with it in $A^{\star}$ and it is also the case for $f(m)$ in $B^{\star}$. By induction hypothesis applied to $A^{\star}[\bullet / X]$ and $B^{\star}[\bullet / X]$, we have $A^{\star}[\bullet / X] \simeq_{f} B^{\star}[\bullet / X]$ and we can conclude $A^{\star} \simeq_{f} B^{\star}$.

- $A^{\star} \simeq_{f} B^{\star} \Rightarrow A \simeq_{\mathcal{E}} B$, we have $A \simeq_{\mathcal{E}} A^{m}$ and $B \simeq_{\mathcal{E}} B^{m}$ by lemma 8 , and this entails with the previous implications: $A^{m \star} \simeq_{f} A^{\star} \simeq_{f} B^{\star} \simeq_{f} B^{m \star}$. By lemma 10 we can deduce $A^{m} \simeq_{\mathcal{E}} B^{m}$ and finally $A \simeq_{\mathcal{E}} B$.

Remark: If, instead of definition 2, we consider polarized isomorphisms given by the composition of proofs of $\vdash ? N^{\perp}, M$ and $\vdash ? M^{\perp}, N$, we obtain the same result since our result about game isomorphisms (theorem 1) applies to any strategy on $A \rightarrow B$ and not only central ones.

\section{Deterministic classical isomorphisms}

As shown by many works $[9,10,15,17]$ deterministic classical systems can be classified into two categories: call-by-name and call-by-value systems. Moreover in each category the different systems are mainly equivalent. To represent these two evaluation paradigms, we will use call-by-name and call-by-value $\lambda \mu$-calculi.

We consider Selinger's extension of the $\lambda \mu$-calculus with disjunction [22]. The $\lambda \mu$-terms are built from two disjoint sets of variables, the $\lambda$-variables $x, y, \ldots$ and the $\mu$-variables $\alpha, \beta, \ldots$ :

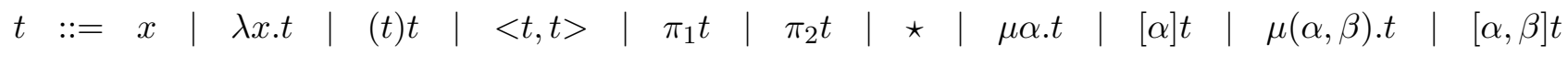

The associated simple types are:

$$
A::=X|A \rightarrow A \quad| A \wedge A \quad|\quad| \quad A \vee A \mid \perp
$$


and a typing judgment has the shape $\Gamma \vdash t: A \mid \Delta$ where $\Gamma$ contains typing declarations for the $\lambda$-variables and $\Delta$ contains typing declarations for the $\mu$-variables. The typing rules are:

$$
\begin{aligned}
& \overline{x: A \vdash x: A \mid} \quad \overline{\Gamma \vdash \star: \top \mid \Delta} \\
& \frac{\Gamma \vdash t: B \mid \Delta}{\Gamma \backslash\{x: A\} \vdash t: A \rightarrow B \mid \Delta} \\
& \frac{\Gamma \vdash t: A \rightarrow B\left|\Delta \quad \Gamma^{\prime} \vdash u: A\right| \Delta^{\prime}}{\Gamma \cup \Gamma^{\prime} \vdash(t) u: B \mid \Delta \cup \Delta^{\prime}} \\
& \frac{\Gamma \vdash t: A|\Delta \quad \Gamma \vdash u: B| \Delta}{\Gamma \vdash<t, u>: A \wedge B \mid \Delta} \\
& \frac{\Gamma \vdash t: A \wedge B \mid \Delta}{\Gamma \vdash \pi_{1} t: A \mid \Delta} \quad \frac{\Gamma \vdash t: A \wedge B \mid \Delta}{\Gamma \vdash \pi_{2} t: B \mid \Delta} \\
& \frac{\Gamma \vdash t: \perp \mid \Delta}{\Gamma \vdash \mu \alpha \cdot t: A \mid \Delta \backslash\{\alpha: A\}} \\
& \frac{\Gamma \vdash t: A \mid \Delta}{\Gamma \vdash[\alpha] t: \perp \mid \Delta \cup\{\alpha: A\}} \\
& \Gamma \vdash t: \perp \mid \Delta \\
& \overline{\Gamma \vdash \mu(\alpha, \beta) . t: A \vee B \mid \Delta \backslash\{\alpha: A, \beta: B\}} \\
& \frac{\Gamma \vdash t: A \vee B \mid \Delta}{\Gamma \vdash[\alpha, \beta] t: \perp \mid \Delta \cup\{\alpha: A, \beta: B\}}
\end{aligned}
$$

If we consider the call-by-name $={ }_{\beta \eta \mu \rho \theta}^{\mathrm{cbn}}$ and the call-by-value $={ }_{\beta \eta \mu \rho \theta}^{\mathrm{cbv}}$ equational theories (defined in the next sections), we obtain the following notions of isomorphisms:

\section{Definition 25 (Isomorphims of types)}

Two types $A$ and $B$ are isomorphic in the call-by-name (resp. call-by-value) $\lambda \mu$-calculus, denoted by $A \simeq_{\lambda \mu}^{\text {cbn }} B$ (resp. $\left.A \simeq_{\lambda \mu}^{\text {cbv }} B\right)$, if there exist two $\lambda \mu$-terms $t$ such that $x: B \vdash t: A \mid$ and $u$ such that $y: A \vdash u: B \mid$ with $(\lambda x . t) u==_{\beta \eta \mu \rho \theta}^{\mathrm{cbn}} y$ (resp. $\left.(\lambda x . t) u={ }_{\beta \eta \mu \rho \theta}^{\mathrm{cbv}} y\right)$ and $(\lambda y . u) t={ }_{\beta \eta \mu \rho \theta}^{\mathrm{cbn}} x$ (resp. $\left.(\lambda y \cdot u) t={ }_{\beta \eta \mu \rho \theta}^{\mathrm{cbv}} x\right)$.

\subsection{Call-by-name isomorphisms}

The call-by-name typed equational theory $={ }_{\beta \eta \mu \rho \theta}^{\mathrm{cbn}}$ of the $\lambda \mu$-calculus [22] is generated by:

$$
\begin{aligned}
& (\lambda x . t) u=\beta \quad t[u / x] \quad: A \\
& \lambda x .(t) x=\eta_{\eta} t \quad: A \rightarrow B \quad x \notin t \\
& \pi_{1}<t, u>\quad=\beta \quad t \quad: A \\
& \pi_{2}<t, u>\quad=\beta \quad u \quad: A \\
& <\pi_{1} t, \pi_{2} t>={ }_{\eta} \quad t \quad: A \wedge B \\
& \star={ }_{\eta} \quad t \quad: \top \\
& (\mu \alpha . t) u={ }_{\mu} \quad \mu \alpha . t\left[[\alpha](v) u /{ }_{[\alpha] v}\right]: A \\
& \pi_{1} \mu \alpha . t=\mu \quad \mu \alpha . t\left[[\alpha] \pi_{1} v /[\alpha] v\right] \quad: A \\
& \pi_{2} \mu \alpha . t=\mu \quad \mu \alpha . t\left[[\alpha] \pi_{2} v /[\alpha] v\right]: A \\
& {[\beta] \mu \alpha . t={ }_{\rho} \quad t[\beta / \alpha] \quad: \perp} \\
& \mu \alpha[\alpha] t={ }_{\theta} \quad t \quad: A \\
& {[\alpha, \beta] \mu \gamma . t=\rho \quad t[[\alpha, \beta] v /[\gamma] v] \quad: \perp} \\
& {\left[\alpha^{\prime}, \beta^{\prime}\right] \mu(\alpha, \beta) . t={ }_{\rho} \quad t\left[\alpha^{\alpha^{\prime}} / \alpha,{ }^{\beta^{\prime}} / \beta\right] \quad: \perp} \\
& \mu(\alpha, \beta)[\alpha, \beta] t={ }_{\theta} \quad t \quad: A \vee B \quad \alpha, \beta \notin t \\
& {[\alpha] t={ }_{\rho} \quad t \quad: \perp}
\end{aligned}
$$

where $\left.t^{C\{v\}} /[\alpha] v\right]$ is obtained by substituting any subterm of $t$ of the shape $[\alpha] v$ by $C\{v\}$ and any subterm of $t$ of the shape $[\alpha, \beta] v$ by $C\{\mu \alpha[\alpha, \beta] v\}$. 
In order to apply our result about polarized isomorphisms of types to the $\lambda \mu$-calculus, we use a translation in LLP.

The translation of the call-by-name $\lambda \mu$-calculus into LLP is obtained by translating types as negative formulas:

$$
\begin{aligned}
X^{-} & =X^{\perp} \\
(A \rightarrow B)^{-} & =! A^{-} \multimap B^{-}=? A^{-\perp} \not B^{-} \\
(A \wedge B)^{-} & =A^{-} \& B^{-} \\
\top^{-} & =\top \\
(A \vee B)^{-} & =A^{-} \ngtr B^{-} \\
\perp^{-} & =\perp
\end{aligned}
$$

the judgment $\Gamma \vdash t: A \mid \Delta$ is translated as $\vdash ?\left(\Gamma^{-}\right)^{\perp}, A^{-}, \Delta^{-}$. The translation of terms is then easy to derive and is given in [15].

\section{Proposition 5 (Simulation)}

If $t$ and $u$ are two $\lambda \mu$-terms such that $t={ }_{\beta \eta \mu \rho \theta}^{\text {cbn }} u$ then $t^{-}={ }_{\beta \eta} u^{-}$.

Definition 26 ( $\mathcal{E}_{n}$-isomorphism)

Let $\mathcal{E}_{n}$ be the equational theory generated by the following equations:

$$
\begin{aligned}
A \wedge B & =B \wedge A \\
A \wedge(B \wedge C) & =(A \wedge B) \wedge C \\
A \wedge \top & =A \\
A \vee B & =B \vee A \\
A \vee(B \vee C) & =(A \vee B) \vee C \\
A \vee \perp & =A \\
A \vee(B \wedge C) & =(A \vee B) \wedge(A \vee C) \\
A \vee \top & =\top \\
(A \wedge B) \rightarrow C & =A \rightarrow(B \rightarrow C) \\
\top \rightarrow A & =A \\
A \rightarrow(B \vee C) & =(A \rightarrow B) \vee C
\end{aligned}
$$

Two types $A$ and $B$ are $\mathcal{E}_{n}$-isomorphic, denoted by $A \simeq_{\mathcal{E}_{n}} B$, if they are equal in the theory $\mathcal{E}_{n}$. 
The theory $\mathcal{E}_{n}(\rightarrow, \wedge, \top)$ is given by:

$$
\begin{aligned}
A \wedge B & =B \wedge A \\
A \wedge(B \wedge C) & =(A \wedge B) \wedge C \\
A \wedge \top & =A \\
(A \wedge B) \rightarrow C & =A \rightarrow(B \rightarrow C) \\
\top \rightarrow A & =A \\
A \rightarrow(B \wedge C) & =(A \rightarrow B) \wedge(A \rightarrow C) \\
A \rightarrow \top & =\top
\end{aligned}
$$

\section{Lemma 11}

The theory $\mathcal{E}_{n}(\rightarrow, \wedge, \top)$ is included in $\mathcal{E}_{n}$.

Proof: We have two equations to prove:

$$
\begin{aligned}
A \rightarrow(B \wedge C) & =A \rightarrow(\perp \vee(B \wedge C)) \\
& =(A \rightarrow \perp) \vee(B \wedge C) \\
& =((A \rightarrow \perp) \vee B) \wedge((A \rightarrow \perp) \vee C) \\
& =(A \rightarrow(\perp \vee B)) \wedge((A \rightarrow \perp) \vee C) \\
& =(A \rightarrow B) \wedge((A \rightarrow \perp) \vee C) \\
& =(A \rightarrow B) \wedge(A \rightarrow(\perp \vee C)) \\
& =(A \rightarrow B) \wedge(A \rightarrow C)
\end{aligned}
$$

and

$$
\begin{aligned}
A \rightarrow \top & =A \rightarrow(\perp \vee \top) \\
& =(A \rightarrow \perp) \vee \top \\
& =\top
\end{aligned}
$$

\section{Proposition 6}

If $A$ and $B$ are two types, $A \simeq_{\lambda \mu}^{\text {cbn }} B \Longleftrightarrow A^{-} \simeq_{\mathrm{LLP}} B^{-} \Longleftrightarrow A \simeq_{\mathcal{E}_{n}} B$.

Proof: The first implication $A \simeq_{\lambda \mu}^{\mathrm{cbn}} B \Rightarrow A^{-} \simeq_{\mathrm{LLP}} B^{-}$is a consequence of proposition 5: if $x: B \vdash t: A \mid$ and $y: A \vdash u: B \mid$ with compositions equal to identity, we obtain two proofs $t^{-}$of $\vdash ? A^{-\perp}, B^{-}$and $u^{-}$of $\vdash ? B^{-\perp}, A^{-}$which are isomorphisms in LLP (see remark after theorem 2).

The second implication $A^{-} \simeq{ }_{\mathrm{LLP}} B^{-} \Rightarrow A \simeq_{\mathcal{E}_{n}} B$ is proved with theorem 2 which implies

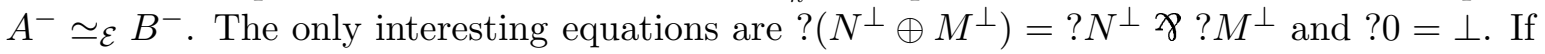
an exponential connective appears in a formula $A^{-}$, it must be a translation of some $C \rightarrow D$ which gives ? $C^{\perp}$ ช8 $D$. In this particular case we have the equation $?\left(C_{1}^{-\perp} \oplus C_{2}^{-\perp}\right)$ ช8 $D^{-}=$ $? C_{1}^{-\perp} \not 2 ? C_{2}^{-\perp} \not \supset D^{-}$which corresponds exactly to $\left(C_{1} \wedge C_{2}\right) \rightarrow D=C_{1} \rightarrow\left(C_{2} \rightarrow D\right)$. For the other equation, it must be applied in the shape ?0 ₹8 $D^{-}=\perp>8 D^{-}$which corresponds to $\top \rightarrow D=D$.

The third implication $A \simeq_{\mathcal{E}_{n}} B \Rightarrow A \simeq_{\lambda \mu}^{\text {cbn }} B$ is just a syntactical verification. 


\section{Corollary 6.1}

If $A$ and $B$ are two types using only the connectives $\rightarrow, \wedge$ and $\top, A \simeq_{\lambda \mu}^{\text {cbn }} B \Longleftrightarrow A \simeq_{\mathcal{E}_{n}(\rightarrow, \wedge, \top)} B$.

Proof: By proposition 6 , we have $A \simeq_{\mathcal{E}_{n}} B$. Up to the equations of $\mathcal{E}_{n}(\rightarrow, \wedge, \top)$, any formula with only $\rightarrow, \wedge$ and $T$ is equal to a formula which is either $T$ or a conjunction of formulas with only $\rightarrow$ (this corresponds to lemma 8 ). We can then verify that two such formulas $A$ and $B$ equal in $\mathcal{E}_{n}$ (or equivalently such that $A^{-} \simeq_{\mathcal{E}} B^{-}$) are equal in $\mathcal{E}_{n}(\rightarrow, \wedge, \top$ ).

The second direction is immediate with proposition 6 and lemma 11.

If isomorphisms in the $\lambda$-calculus are considered up to usual $\beta \eta$-equivalence, we have:

\section{Corollary 6.2}

If $A$ and $B$ are two types using only the connectives $\rightarrow, \wedge$ and $\top$ then $A \simeq_{\lambda} B \Longleftrightarrow A \simeq_{\lambda \mu}^{\text {cbn }} B$.

Proof: Since the $\lambda$-calculus is a subsystem of the $\lambda \mu$-calculus any type isomorphism of the $\lambda$ calculus is an isomorphism of the $\lambda \mu$-calculus. In the other direction we just verify, with corollary 6.1 , that the theory $\mathcal{E}_{n}(\rightarrow, \wedge, \top)$ is validated by the $\lambda$-calculus.

This gives a new proof of the equational theory of the isomorphisms of types for the $\lambda$ calculus $[23,12]$.

\subsection{Call-by-value isomorphisms}

Values are particular $\lambda \mu$-terms defined by the following grammar:

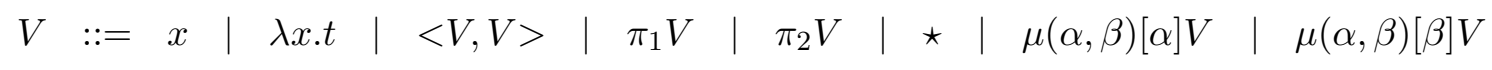

with $\alpha, \beta \notin V$.

The call-by-value typed equational theory $=_{\beta \eta \mu \rho \theta}^{\mathrm{cbv}}$ of the $\lambda \mu$-calculus [22] is generated by:

$$
\begin{aligned}
& (\lambda x . t) V={ }_{\beta} \quad t\left[{ }^{V} / x\right] \quad: A \\
& \lambda x .(V) x={ }_{\eta} V \quad: A \rightarrow B \quad x \notin V \\
& \pi_{1}<V, W>\quad=\beta \quad V \quad: A \\
& \pi_{2}<V, W>=\beta \quad W \quad: A \\
& <\pi_{1} V, \pi_{2} V>={ }_{\eta} \quad V \quad: A \wedge B \\
& \star={ }_{\eta} V \quad: \top \\
& (\lambda x . x) t={ }_{\beta} \quad t \quad: A \\
& (\lambda y . u)(\lambda x . t) v={ }_{\beta} \quad(\lambda x .(\lambda y . u) t) v \quad: A \quad x \notin u \\
& (\lambda x .(\lambda y .(x) y) t) u=\beta \quad(u) t \quad: A \quad x \notin t \\
& (\lambda x .(\lambda y .<x, y>) t) u \quad=\beta \quad<u, t>\quad: A \wedge B \quad x \notin t \\
& \left(\lambda x . \pi_{1} x\right) t=\beta \quad \pi_{1} t \quad: A \\
& \left(\lambda x . \pi_{2} x\right) t=\pi_{2} \quad: A \\
& (\lambda x . t) \mu \alpha . u={ }_{\mu} \quad \mu \alpha . u\left[^{(\lambda x \cdot[\alpha] t) v}{ }_{[\alpha] v]}\right]: A \quad \alpha \notin t \\
& {[\beta] \mu \alpha . t={ }_{\rho} \quad t[\beta / \alpha] \quad: \perp} \\
& \mu \alpha[\alpha] t={ }_{\theta} \quad t \quad: A \quad \alpha \notin t \\
& {\left[\alpha^{\prime}, \beta^{\prime}\right] \mu(\alpha, \beta) \cdot t={ }_{\rho} \quad t\left[\alpha^{\alpha^{\prime}} / \alpha,{ }^{\beta^{\prime}} / \beta\right] \quad: \perp} \\
& \mu(\alpha, \beta)[\alpha, \beta] t={ }_{\theta} \quad t \quad: A \vee B \quad \alpha, \beta \notin t \\
& {[\alpha] t={ }_{\rho} \quad t \quad: \perp} \\
& (\lambda x \cdot[\alpha] x) t={ }_{\beta} \quad[\alpha] t \quad: \perp \\
& (\lambda x \cdot[\alpha, \beta] x) t=\beta \quad[\alpha, \beta] t \quad: \perp
\end{aligned}
$$


where $V$ and $W$ are values.

The translation of the call-by-value $\lambda \mu$-calculus into LLP is obtained by translating types as positive formulas:

$$
\begin{aligned}
X^{+} & =X \\
(A \rightarrow B)^{+} & =!\left(A^{+} \multimap ? B^{+}\right)=!\left(A^{+\perp} \text { ช } ? B^{+}\right) \\
(A \wedge B)^{+} & =A^{+} \otimes B^{+} \\
\top^{+} & =1 \\
(A \vee B)^{+} & =A^{+} \oplus B^{+} \\
\perp^{+} & =0
\end{aligned}
$$

the judgment $\Gamma \vdash t: A \mid \Delta$ is translated as $\vdash\left(\Gamma^{+}\right)^{\perp}, ? A^{+}, ? \Delta^{+}$. The translation of terms is then easy to derive and is given in [15].

\section{Proposition 7 (Simulation)}

If $t$ and $u$ are two $\lambda \mu$-terms such that $t={ }_{\beta \eta \mu \rho \theta}^{\mathrm{cbv}} u$ then $t^{+}={ }_{\beta \eta} u^{+}$.

\section{Definition 27 ( $\mathcal{E}_{v}$-isomorphism)}

Let $\mathcal{E}_{v}$ be the equational theory generated by the following equations:

$$
\begin{aligned}
A \wedge B & =B \wedge A \\
A \wedge(B \wedge C) & =(A \wedge B) \wedge C \\
A \wedge \top & =A \\
A \vee B & =B \vee A \\
A \vee(B \vee C) & =(A \vee B) \vee C \\
A \vee \perp & =A \\
A \wedge(B \vee C) & =(A \wedge B) \vee(A \wedge C) \\
A \wedge \perp & =\perp \\
(A \vee B) \rightarrow C & =(A \rightarrow C) \wedge(B \rightarrow C) \\
\perp \rightarrow A & =\top
\end{aligned}
$$

Two types $A$ and $B$ are $\mathcal{E}_{v}$-isomorphic, denoted by $A \simeq_{\mathcal{E}_{v}} B$, if they are equal in the theory $\mathcal{E}_{v}$.

The theory $\mathcal{E}_{v}(\rightarrow, \wedge, \top)$ is given by:

$$
\begin{aligned}
A \wedge B & =B \wedge A \\
A \wedge(B \wedge C) & =(A \wedge B) \wedge C \\
A \wedge \top & =A
\end{aligned}
$$

Remark: In particular, $A \rightarrow(B \rightarrow C) \not \neg B \rightarrow(A \rightarrow C)$ because $\lambda x . \lambda y$. $(f) x y \neq f$ in call-by-value.

\section{Proposition 8}

If $A$ and $B$ are two types, $A \simeq_{\lambda \mu}^{\text {cbv }} B \Longleftrightarrow A^{+} \simeq{ }_{\mathrm{LLP}} B^{+} \Longleftrightarrow A \simeq_{\mathcal{E}_{v}} B$. 
Proof: The first implication $A \simeq_{\lambda \mu}^{\mathrm{cbv}} B \Rightarrow A^{+} \simeq_{\mathrm{LLP}} B^{+}$is a consequence of proposition 5: if $x: B \vdash t: A \mid$ and $y: A \vdash u: B \mid$ with compositions equal to identity, we obtain two proofs $t^{+}$of $\vdash A^{+\perp}, ? B^{+}$and $u^{+}$of $\vdash B^{+\perp}, ? A^{+}$which are isomorphisms in LLP (see remark after theorem 2).

The second implication $A^{+} \simeq$ LLP $B^{+} \Rightarrow A \simeq_{\mathcal{E}_{v}} B$ is proved with theorem 2 which implies $A^{+^{\star}} \simeq_{f} B^{+^{\star}}$. In the spirit of lemmas 8 and 10 , we can show that any type is equal in $\mathcal{E}_{v}$ to a type with the connectives $\vee$ and $\perp$ appearing only in head position, and two such types corresponding to isomorphic forests are equal in $\mathcal{E}_{v}$.

The third implication $A \simeq_{\mathcal{E}_{v}} B \Rightarrow A \simeq_{\lambda \mu}^{\text {cbv }} B$ is just a syntactical verification.

\section{Corollary 8.1}

If $A$ and $B$ are two types using only the connectives $\rightarrow, \wedge$ and $\top, A \simeq_{\lambda \mu}^{\text {cbv }} B \Longleftrightarrow A \simeq_{\mathcal{E}_{v}(\rightarrow, \wedge, \top)} B$.

Proof: By theorem 2, we have $A^{+^{\star}} \simeq_{f} B^{+^{\star}}$ and we can show that two types based only on $\rightarrow$, $\wedge$ and $\top$ with the same associated forest are equal in $\mathcal{E}_{v}(\rightarrow, \wedge, \top)$ as for lemma 10.

The other direction is immediate with proposition 8.

The equational theory for the call-by-value $\lambda$-calculus [20] (or $\lambda_{v}$-calculus) is given by:

$$
\begin{array}{rllll}
(\lambda x . t) V & ={ }_{\beta} & t[V / x] & : A & \\
\lambda x .(V) x & ={ }_{\eta} & V & : A \rightarrow B & x \notin V \\
\pi_{1}<V, W> & ={ }_{\beta} & V & : A & \\
\pi_{2}<V, W> & ={ }_{\beta} & W & : A & \\
<\pi_{1} V, \pi_{2} V> & ={ }_{\eta} & V & : A \wedge B \\
\star & ={ }_{\eta} & V & : \top &
\end{array}
$$

\section{Corollary 8.2}

If $A$ and $B$ are two types using only the connectives $\rightarrow, \wedge$ and $\top, A \simeq_{\lambda_{v}} B \Longleftrightarrow A \simeq_{\lambda \mu}^{\text {cbv } B}$.

Proof: The call-by-value $\lambda$-calculus is a subsystem of the call-by-value $\lambda \mu$-calculus and that gives the first implication. In the other direction we just verify that the theory $\mathcal{E}_{v}(\rightarrow, \wedge, \top)$ is validated by the $\lambda_{v}$-calculus.

\section{Classical and intuitionistic disjunctions}

In the previous sections, we have been able to give a finite axiomatization of the theory of isomorphisms of types for the call-by-name $\lambda \mu$-calculus with disjunction $(\vee)$ and contradiction $(\perp)$ types. However, Balat, Di Cosmo and Fiore [13] have shown that, in the $\lambda$-calculus case, this theory is not finitely axiomatizable. We are going to discuss this seeming contradiction.

The equational theory of the call-by-name $\lambda \mu$-calculus for the language corresponding to types $\rightarrow \wedge$ and $T$ is a conservative extension of the equational theory of the $\lambda$-calculus for the same types. When we go to the disjunction case, this is not true anymore. The equational theory of the $\lambda$-calculus with disjunction corresponding to the axioms of bicartesian closed categories contains:

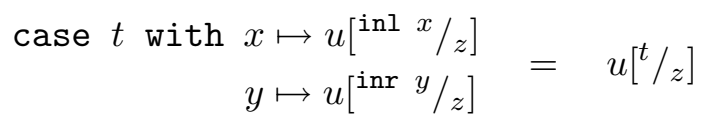


which is used to prove the isomorphism $(A \vee B) \rightarrow C \simeq(A \rightarrow C) \wedge(B \rightarrow C)$ (wrong in the call-by-name $\lambda \mu$-calculus). If we consider the two main particular cases of (1): $u=z$ and $t=z$, we obtain for $u=z$ :

$$
\text { case } \begin{aligned}
t \text { with } x & \mapsto \operatorname{inl} x \\
y & \mapsto \text { inr } y
\end{aligned}=t
$$

This equation is validated in the $\lambda \mu$-calculus and corresponds to:

$$
\mu \gamma[\gamma](\lambda y . \mu(\alpha, \beta)[\beta] y) \mu \beta[\gamma](\lambda x . \mu(\alpha, \beta)[\alpha] x) \mu \alpha[\alpha, \beta] t=t
$$

with $\alpha, \beta \notin t$. But with $t=z$ in (1):

$$
\text { case } z \text { with } x \mapsto u\left[\begin{array}{ll}
\text { inl } x / z] \\
y & \mapsto u\left[\begin{array}{ll}
\text { inr } & y / z
\end{array}\right]=u
\end{array}\right.
$$

and this equation is not realized in the $\lambda \mu$-calculus.

From a categorical point of view, the notion of control category [22] which gives the models of the call-by-name $\lambda \mu$-calculus is based on a disjunction which is a binoidal functor and not a bifunctor. Selinger proved that a control category with a bifunctorial disjunction is a boolean algebra and we are back to section 1 . This shows that, in some sense, the theories of the $\lambda$-calculus with disjunction and of the $\lambda \mu$-calculus with disjunction are incompatible.

This explains how our result is not comparable with Balat-Di Cosmo-Fiore's one, in particular the disjunction distributes over the conjunction here and this is the converse in their setting. The only result we can deduce about the $\lambda$-calculus from ours are the equations for the isomorphisms of types of the $\lambda$-calculus with a constrained disjunction which verifies equation (2) but not equation (1).

\section{Tarski's problem}

The question of isomorphisms of types consists in finding some equational characterization of the isomorphisms of a given logical system, whereas Tarski's problem consists in proving that some equational theory characterizes the equality in some number structure (natural numbers with selected operations:,$+ \times, \ldots$, real numbers, $\ldots)$.

Composing these two questions, we can try to find number structures and logical systems such that equality in the first one corresponds to isomorphisms of types in the second one. The key result of this kind is given by theorem 3 and an interesting use of such a correspondence is given in $[13]$.

We are going to describe the relations between polarized isomorphisms and the associated number models. As a consequence, these results give also relations with call-by-name and call-byvalue classical isomorphisms.

\section{Definition 28 (Tarski model of isomorphisms)}

Let $\mathcal{S}$ be a logical system, a Tarski model of the isomorphisms of $\mathcal{S}$ is a model of the equational theory corresponding to the isomorphisms of types of $\mathcal{S}$ which doesn't validate any other universally closed equation.

\section{Theorem 3 (Soloviev [23])}

$\left(\mathbb{N}, \cdot, 1,(-)^{-}\right)$is a Tarski model of the isomorphisms of the simply typed $\lambda$-calculus with the connectives $(\rightarrow, \wedge, \top)$. 


\section{Corollary 3.1}

$\left(\mathbb{N}, \cdot, 1,(-)^{-}\right)$is a Tarski model of the isomorphisms of the simply typed call-by-name $\lambda \mu$-calculus with the connectives $(\rightarrow, \wedge, \top)$.

Proof: We have shown in corollary 6.2 that the equational theory for the isomorphisms of types of the call-by-name $\lambda \mu$-calculus and of the $\lambda$-calculus are the same for the language $(\rightarrow, \wedge, \top)$.

We are going to use Macintyre's result on Schanuel's conjecture and exponential rings [18] to give a sufficient condition to be a Tarski model of the positive isomorphisms of LLP. By duality, this gives also models of the negative isomorphisms and, as a consequence, models of call-by-name and call-by-value classical isomorphisms.

We recall some definitions about exponential rings [24] with the slightly more general case of exponential semi-rings.

\section{Definition 29 (Semi-ring)}

A semi-ring is a tuple $(R,+, 0, \cdot, 1)$ such that $(R,+, 0)$ is a commutative monoid and $(R, \cdot, 1)$ is a monoid (if it is commutative, the semi-ring is commutative), and moreover:

$$
\begin{aligned}
x \cdot(y+z) & =(x \cdot y)+(x \cdot z) \\
(y+z) \cdot x & =(y \cdot x)+(z \cdot x) \\
x \cdot 0 & =0 \\
0 \cdot x & =0
\end{aligned}
$$

\section{Definition 30 (Exponential semi-ring)}

An exponential semi-ring $(R,+, 0, \cdot, 1, E)$ is a commutative semi-ring $(R,+, 0, \cdot, 1)$ with a map $E$ from $R$ to $R$ such that:

$$
\begin{aligned}
E(x+y) & =E(x) \cdot E(y) \\
E(0) & =1
\end{aligned}
$$

so that $E$ is a morphism of monoid from $(R,+, 0)$ to $(R, \cdot, 1)$.

The theory of exponential semi-rings is equational and coincides with the theory of polarized isomorphisms written in a purely positive language:

$\begin{array}{ccc}A \otimes B & = & B \otimes A \\ (A \otimes B) \otimes C & = & A \otimes(B \otimes C) \\ A \otimes 1 & = & A \\ A \oplus B & = & B \oplus A \\ (A \oplus B) \oplus C & = & A \oplus(B \oplus C) \\ A \oplus 0 & = & A \\ A \otimes(B \oplus C) & = & (A \otimes B) \oplus(A \otimes C) \\ A \otimes 0 & = & 0 \\ \neg(A \oplus B) & = & \neg A \otimes \neg B \\ \neg 0 & = & 1\end{array}$

where $\neg P$ stands for $! P^{\perp}$. We just interpret $\oplus$ by,+ 0 by $0, \otimes$ by $\cdot, 1$ by 1 and $\neg$ by $E$.

Definition 31 (Exponential ring)

An exponential semi-ring $R$ is an exponential ring if the underlying semi-ring is a ring. 
Definition 32 (Schanuel's condition)

Let $R$ be an exponential ring which is a domain and has characteristic $0, R$ satisfies $S$ chanuel's condition if for any $\alpha_{1}, \ldots, \alpha_{n}$ in $R$ linearly independent over $\mathbb{Q}$, the ring $\mathbb{Z}\left[\alpha_{1}, \ldots, \alpha_{n}, E\left(\alpha_{1}\right), \ldots, E\left(\alpha_{n}\right)\right]$ has transcendence degree at least $n$ over $\mathbb{Z}$.

Theorem 4 (Macintyre [18])

If $R$ is an exponential ring which satisfies Schanuel's condition, if $e_{1}=e_{2}$ is a closed equation in the language $(+, 0, \cdot, 1, E)$ true in $R$, it is also true in the free exponential ring on 0 generators.

\section{Corollary 4.1}

If $R$ is an exponential ring which satisfies Schanuel's condition, $R$ is a Tarski model of the positive isomorphisms of LLP.

In the particular case of the exponential $\operatorname{ring}(\mathbb{R},+, 0, \cdot, 1, e)$, the fact that $\mathbb{R}$ satisfies Schanuel's condition is called Schanuel's conjecture.

\section{Corollary 4.2}

If Schanuel's conjecture is true, $(\mathbb{R},+, 0, \cdot, 1, e)$ is a Tarski model of the positive isomorphisms of LLP.

The terminology for the linear connectives given by Girard is the following: $\otimes, \not 2,1$ and $\perp$ are the multiplicative connectives, $\oplus, \&, 0$ and $\top$ are the additive connectives and ! and ? are the exponential connectives. This originally came from linear algebra and from the key isomorphism of LL: $(A \& B)=! A \otimes ! B$. We somehow give here a concrete interpretation of these ideas by really interpreting the linear connectives by the corresponding operations on real numbers: additives by + , multiplicatives by $\cdot$ and exponentials by $e$.

Macintyre's result gives a sufficient condition for having a Tarski model. Concerning a necessary condition we can just make a few remarks. A Tarski model of the positive isomorphisms of LLP must be an exponential semi-ring $S$ and its characteristic must be 0 . Otherwise there exists some $p$ such that $\underbrace{1+\cdots+1}_{p}=0$, this gives an equation which is not valid in LLP. We can always move to the case of rings by introducing the free exponential ring $R$ generated by $S$. If this ring doesn't satisfies Schanuel's condition, it must at least verify that $E(1), E(E(1)), E(E(E(1))), \ldots$ are transcendental otherwise from a polynomial $P$ such that $P\left(E^{n}(1)\right)=0$ we can derive an equation on the language $(+, 0, \cdot, 1, E)$ by decomposing $P=P_{1}-P_{2}$ with $P_{1}$ and $P_{2}$ in this language: $P_{1}\left(E^{n}(1)\right)=P_{2}\left(E^{n}(1)\right)$ which is not valid in LLP. In particular if $(\mathbb{R},+, 0, \cdot, 1, e)$ is a Tarski model of LLP, $e^{e}$ must be transcendental and this is an open problem in number theory.

Remark: If we look at the translation of the call-by-value implication $A \rightarrow B$ in LLP we obtain $!(A \multimap ? B)=!\left(A^{\perp} \not \gamma ? B\right)$. It seems natural to interpret it by $B^{A}=e^{A \ln B}$ but ? transforms $\oplus$ into 8 while $\ln$ transforms $\cdot$ into + and we can't see any use for this.

Assuming Schanuel's conjecture, $\mathbb{R}$ is a Tarski model of the isomorphisms of LLP, this entails that we can associate with any equation on real numbers in the language $(+, 0, \cdot, 1, e)$, an algorithmic interpretation by computing the proofs in LLP corresponding to this isomorphism (or the corresponding $\lambda \mu$-terms). 


\section{Further work}

We have shown how a game semantics approach allows to characterize classical isomorphisms of types (and as a consequence to give a new proof for the intuitionistic ones) in a propositional setting. Previous syntactical works [12] allowed to give results on polymorphic systems. We can wonder if it is possible to extend our game approach to the second order setting.

An advantage of game semantics is the possibility of modeling not only the $\lambda$-calculus and other logical systems but also programming primitives of various kinds: non determinism [14], ground type references [3], general references [1], ... The key result of our approach is theorem 1 and if we look at the proof we can see that we only use determinism and visibility of strategies and from a computational viewpoint this corresponds to the game model of Idealized Algol (IA) [3]. As a consequence this theorem gives a characterization of the isomorphisms of types for IA. However we use the visibility condition so that our result cannot be directly applied to general references and we can wonder if it is possible to remove this constraint in our proof to extend the result.

A more general question than isomorphisms of types is the characterization of retractions in a given logical system. This is known to be a difficult problem for the $\lambda$-calculus [19]. We can try to apply game semantics ideas to address this problem.

Another related question is the study of isomorphisms of types in usual Linear Logic. Balat and Di Cosmo have given a syntactical characterization of the isomorphisms of types in MLL [6] (the multiplicative fragment of Linear Logic). The question of the extension of this result to richer fragments of LL remains open. We must precise that our complete characterization of polarized isomorphisms cannot be directly of help for the LL problem which is a strongly more general one. However we could hope to apply game semantics, as we have done here, in a non polarized setting. For example MLL game semantics [2] might be use to give an alternative proof of Balat and Di Cosmo's result. The extension to richer fragments is once again problematic since we still don't know if game models are precise enough for these fragments. Two possible fragments to look at are the MALL case for which Abramsky and Melliès' model [4] might be used and the MELL case with Baillot-Danos-Ehrhard-Regnier's model [5].

Thanks to V. Danos for his suggestions on a previous game model that led to this work, and thanks to V. Balat and R. Di Cosmo for having introduced me to the question of isomorphisms of types.

\section{References}

[1] Samson Abramsky, Kohei Honda, and Guy McCusker. A fully abstract game semantics for general references. In Proceedings of the thirteenth annual symposium on Logic In Computer Science, pages 334-344, Indianapolis, June 1998. IEEE, IEEE Computer Society Press.

[2] Samson Abramsky and Radha Jagadeesan. Games and full completeness for multiplicative linear logic. Journal of Symbolic Logic, 59(2):543-574, June 1994.

[3] Samson Abramsky and Guy McCusker. Full abstraction for idealized algol with passive expressions. Theoretical Computer Science, 227:3-42, September 1999.

[4] Samson Abramsky and Paul-André Melliès. Concurrent games and full completeness. In Proceedings of the fourteenth annual symposium on Logic In Computer Science, pages 431442, Trento, July 1999. IEEE, IEEE Computer Society Press. 
[5] Patrick Baillot, Vincent Danos, Thomas Ehrhard, and Laurent Regnier. Believe it or not, AJM's games model is a model of classical linear logic. In Proceedings of the twelfth annual symposium on Logic In Computer Science, pages 68-75, Warsaw, June 1997. IEEE, IEEE Computer Society Press.

[6] Vincent Balat and Roberto Di Cosmo. A linear logical view of linear type isomorphisms. In Jörg Flum and Mario Rodríguez-Artalejo, editors, Computer Science Logic, volume 1683 of Lecture Notes in Computer Science, pages 250-265. Springer, 1999.

[7] Gérard Berry and Pierre-Louis Curien. Sequential algorithms on concrete data structures. Theoretical Computer Science, 20:265-321, 1982.

[8] Kim Bruce, Roberto Di Cosmo, and Guiseppe Longo. Provable isomorphisms of types. Mathematical Structures in Computer Science, 2(2):231-247, 1992. Proceedings of Symposium on Symbolic Computation, ETH, Zurich, March 1990.

[9] Vincent Danos, Jean-Baptiste Joinet, and Harold Schellinx. LKQ and LKT: Sequent calculi for second order logic based upon dual linear decompositions of classical implication. In JeanYves Girard, Yves Lafont, and Laurent Regnier, editors, Advances in Linear Logic, volume 222 of London Mathematical Society Lecture Note Series, pages 211-224. Cambridge University Press, 1995.

[10] Vincent Danos, Jean-Baptiste Joinet, and Harold Schellinx. A new deconstructive logic: linear logic. Journal of Symbolic Logic, 62(3):755-807, September 1997.

[11] Mariangiola Dezani-Ciancaglini. Characterization of normal forms possessing an inverse in the $\lambda \beta \eta$-calculus. Theoretical Computer Science, 2:323-337, 1976.

[12] Roberto Di Cosmo. Isomorphisms of Types. Progress in Theoretical Computer Science. Birkhäuser, 1995.

[13] Marcelo Fiore, Roberto Di Cosmo, and Vincent Balat. Remarks on isomorphisms in typed lambda calculi with empty and sum types. In Proceedings of the seventeenth annual symposium on Logic In Computer Science, pages 147-156, Copenhagen, July 2002. IEEE, IEEE Computer Society Press.

[14] Russel Harmer. Games and Full Abstraction for Nondeterministic Languages. Ph.D. thesis, Imperial College and University of London, 1999.

[15] Olivier Laurent. Etude de la polarisation en logique. Thèse de doctorat, Université Aix-Marseille II, March 2002. Available at http://www.pps.jussieu.fr/ laurent/these.ps.gz .

[16] Olivier Laurent. Syntax vs. semantics: a polarized approach. Prépublication électronique PPS//03/04//nº 17 (pp), Laboratoire Preuves, Programmes et Systèmes, March 2003. Submitted for publication.

[17] Paul Blain Levy. Call-by-push-value: A subsuming paradigm. In Jean-Yves Girard, editor, Typed Lambda Calculi and Applications '99, volume 1581 of Lecture Notes in Computer Science, pages 228-242. Springer, April 1999. 
[18] Angus Macintyre. Schanuel's conjecture and free exponential rings. Annals of Pure and Applied Logic, 51:241-246, 1991.

[19] Vincent Padovani. Retracts in simple types. In Samson Abramsky, editor, Typed Lambda Calculi and Applications '01, volume 2044 of Lecture Notes in Computer Science, pages 376384. Springer, May 2001.

[20] Gordon Plotkin. Call-by-name, call-by-value and the $\lambda$-calculus. Theoretical Computer Science, 1:125-159, 1975.

[21] Mikael Rittri. Using types as search keys in function libraries. Journal of Functional Programming, 1(1):71-89, 1991.

[22] Peter Selinger. Control categories and duality: on the categorical semantics of the lambda-mu calculus. Mathematical Structures in Computer Science, 11(2):207-260, April 2001.

[23] Sergei Soloviev. The category of finite sets and cartesian closed categories. Journal of Soviet Mathematics, 22(3):1387-1400, 1983.

[24] Lou van den Dries. Exponential rings, exponential polynomials and exponential functions. Pacific Journal of Mathematics, 113(1):51-66, 1984. 